\title{
Three-body decay of ${ }^{6} \mathrm{Be}$
}

\author{
L. V. Grigorenko, ${ }^{1,2,3}$ T. D. Wiser, ${ }^{4}$ K. Mercurio, ${ }^{4}$ R. J. Charity,${ }^{5}$ R. Shane, ${ }^{4}$ L. G. Sobotka, ${ }^{4,5}$ J. M. Elson, ${ }^{5}$ A. H. Wuosmaa, ${ }^{6}$ \\ A. Banu, ${ }^{7}$ M. McCleskey, ${ }^{7}$ L. Trache, ${ }^{7}$ R. E. Tribble, ${ }^{7}$ and M. V. Zhukov ${ }^{8}$ \\ ${ }^{1}$ Flerov Laboratory of Nuclear Reactions, JINR, RU-141980 Dubna, Russia \\ ${ }^{2}$ Gesellschaft für Schwerionenforschung mbH, Plankstrasse 1, D-64291 Darmstadt, Germany \\ ${ }^{3}$ RRC “The Kurchatov Institute," Kurchatov sq. 1, RU-123182 Moscow, Russia \\ ${ }^{4}$ Department of Physics, Washington University, St. Louis, Missouri 63130, USA \\ ${ }^{5}$ Department of Chemistry, Washington University, St. Louis, Missouri 63130, USA \\ ${ }^{6}$ Department of Physics, Western Michigan University, Kalamazoo, Michigan 49008, USA \\ ${ }^{7}$ Cyclotron Institute, Texas A\&M University, College Station, Texas 77843, USA \\ ${ }^{8}$ Fundamental Physics, Chalmers University of Technology, S-41296 Göteborg, Sweden
}

(Received 10 March 2009; published 8 September 2009)

\begin{abstract}
Three-body correlations for the ground-state decay of the lightest two-proton emitter ${ }^{6} \mathrm{Be}$ are studied both theoretically and experimentally. Theoretical studies are performed in a three-body hyperspherical-harmonics cluster model. In the experimental studies, the ground state of ${ }^{6} \mathrm{Be}$ was formed following the $\alpha$ decay of a ${ }^{10} \mathrm{C}$ beam inelastically excited through interactions with Be and $\mathrm{C}$ targets. Excellent agreement between theory and experiment is obtained demonstrating the existence of complicated correlation patterns that can elucidate the structure of ${ }^{6} \mathrm{Be}$ and, possibly, of the $A=6$ isobars.
\end{abstract}

DOI: $10.1103 /$ PhysRevC.80.034602

PACS number(s): 23.50.+z, 23.20.En, 21.60.Gx, 27.20.+n

\section{INTRODUCTION}

Two-proton $(2 p)$ radioactivity was predicted by V. I. Goldansky in 1960 [1] as an exclusively quantum-mechanical phenomenon. True three-body decay, in his terms, is a situation where the sequential emission of the particles is energetically prohibited and all the final-state fragments are emitted simultaneously. These energy conditions are illustrated for ${ }^{6} \mathrm{Be}$ in Fig. 1 that shows that the ${ }^{5} \mathrm{Li}$ ground state (g.s.) is not fully accessible for sequential decay. Because the experimental discovery of ${ }^{45} \mathrm{Fe}$ two-proton radioactivity in $2002[2,3]$, this field has made fast progress. New cases of $2 p$ radioactivity were found for ${ }^{54} \mathrm{Zn}$ [4], ${ }^{19} \mathrm{Mg}$ [5], and, maybe, ${ }^{48} \mathrm{Ni}$ [6]. The $2 p$ correlations were recently measured in ${ }^{45} \mathrm{Fe}$ [7], ${ }^{19} \mathrm{Mg}[5,8],{ }^{16} \mathrm{Ne}[8]$, and ${ }^{10} \mathrm{C}[9]$. An interesting [10], but controversial $[11,12]$, case is the possibility of $2 p$ emission from the deformed isomeric state in ${ }^{94} \mathrm{Ag}$. All these decays exhibit complex correlation patterns. It is argued that studies of these patterns could provide important information about the structure of the decaying nuclei.

With this active research as the background, there is one case that has been unduly forgotten. The ${ }^{6} \mathrm{Be}$ nucleus is the lightest true two-proton emitter in the sense of Goldansky. As this is expected to be the simplest case (smallest Coulomb interaction, expressed cluster structure with closed-shell core), a full understanding of its physics would provide a reliable basis for all future studies of $2 p$ decay. However, until now, theoretical work on ${ }^{6} \mathrm{Be}$ was limited to predicting the energies and widths of its states. In addition, precise experimental data on correlations did not exist. The last experimental work dedicated to correlations in the ${ }^{6} \mathrm{Be}$ g.s. is about 20 years old [13].

There is one more aspect that makes the ${ }^{6} \mathrm{Be}$ case of special interest. In the past decade, large efforts have been directed to studies of ${ }^{6} \mathrm{He}$ with special emphasis to the understanding of the halo properties in this comparatively simple and accessible case. The associated literature comprises hundreds of titles.
To deduce the correlations in the neutron halo in many cases, one has to excite (e.g., Coulex) or destroy (e.g., knockout reactions) this nucleus. Therefore, the interpretation of the experimental data is influenced by the need to clarify details of the reaction mechanism. However, information about the mirror system can be obtained without all this "violence." The isobaric analog state in ${ }^{6} \mathrm{Be}$ decays to the $\alpha+p+p$ channel all by itself, providing the differential data on correlations. These data can be used directly to elucidate the structure of ${ }^{6} \mathrm{Be}$ and possibly of the whole $A=6(T=1)$ isobar. Thus an important opportunity exists for a better understanding of ${ }^{6} \mathrm{He}$ properties through detailed studies of ${ }^{6} \mathrm{Be}$. This has not been exploited previously.

In this work, we provide detailed theoretical calculations of the three-body decay characteristics of ${ }^{6} \mathrm{Be}$ in a three-body cluster $\alpha+p+p$ model. We demonstrate that, in certain aspects, ${ }^{6} \mathrm{Be}$ may be a preferable tool for studies of the $A=6$ isobars, especially considering the high sensitivity of observables to the details of the theoretical models. We then discuss previous experimental and theoretical works on ${ }^{6} \mathrm{Be}$. Subsequently, we report on an experiment where ${ }^{6} \mathrm{Be}$ fragments are formed after the $\alpha$ decay of ${ }^{10} \mathrm{C}$ projectiles excited by inelastic scattering. These data cover the complete kinematic space accessible for three-body decay and the correlations are compared to the theoretical predictions. A brief account of our results was provided in letter form [14], where the ${ }^{6} \mathrm{Be}$ data on complete $2 p$ correlations were also compared to analogous data from the $2 p$ decay of ${ }^{45} \mathrm{Fe}$.

The $\hbar=c=1$ system of units is used in this work. The following notations are used: $E_{T}$ is the system energy and $E_{3 r}$ is the three-body resonance energy both relative to the three-body $\alpha+p+p$ threshold.

\section{THEORETICAL MODEL}

The theoretical framework of this article is largely the same as that developed for the two-proton radioactivity and 


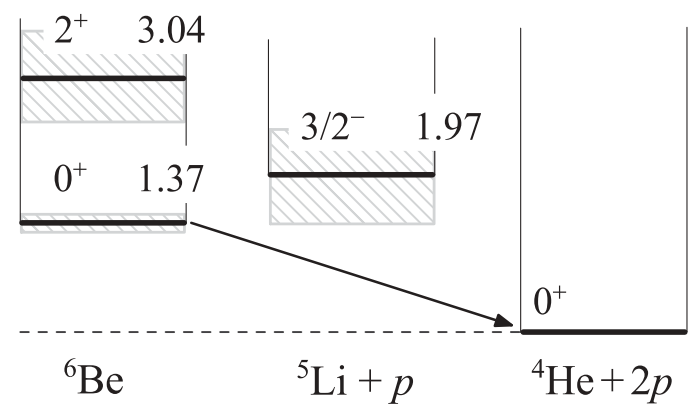

FIG. 1. The energy levels and the decay scheme for ${ }^{6} \mathrm{Be}$ [15]. The ${ }^{6} \mathrm{Be}$ g.s. is a true two-proton emitter in the sense of Goldansky: the sequential decay of this state is not possible as the lowest possible intermediate, the ${ }^{5} \mathrm{Li}$ g.s., is not energetically accessible.

three-body decay studies in Refs. [16-24]. It is based on the three-body cluster model using the hyperspherical-harmonics (HH) method. The predictions obtained with this approach were found to be in very good agreement with experimental widths and momentum distributions $[5,7,8]$.

In this section, we sketch the necessary formalism emphasizing only the points that differ from previous treatments.

\section{A. Hyperspherical harmonics method}

For narrow states, the time-dependent wave function (WF) in a finite domain can be parameterized as

$$
\Psi_{3}^{(+)}\left(\rho, \Omega_{\rho}, t\right)=e^{-\frac{\Gamma}{2} t-i E t} \Psi_{3}^{(+)}\left(\rho, \Omega_{\rho}\right) .
$$

The WF is a function of the hyper-radius $\rho$, the time $t$, and $\Omega_{\rho}$ that specifies all the orientation degrees of freedom. The radial part of this WF can be obtained with good precision as a solution of the inhomogeneous system of equations

$$
\begin{aligned}
& \left(\hat{H}-E_{3 r}\right) \Psi_{E}^{(+)}\left(\rho, \Omega_{\rho}\right)=-i(\Gamma / 2) \Psi_{\text {box }}\left(\rho, \Omega_{\rho}\right), \\
& \hat{H}=\hat{T}+\hat{V}_{c p}\left(\mathbf{r}_{c p_{1}}\right)+\hat{V}_{c p}\left(\mathbf{r}_{c p_{2}}\right)+\hat{V}_{p p}\left(\mathbf{r}_{p_{1} p_{2}}\right) .
\end{aligned}
$$

Here $\Psi_{\text {box }}$ and $E_{3 r}$ are the eigenfunction and the eigenvalue of the equation

$$
(\hat{H}-E) \Psi_{\text {box }}\left(\rho, \Omega_{\rho}\right)=0,
$$

solved with a "box" boundary condition at large $\rho$. The hyperspherical coordinates are defined via the Jacobi vectors

$$
\begin{aligned}
\mathbf{X} & =\mathbf{r}_{p_{1}}-\mathbf{r}_{p_{2}}, \quad \mathbf{Y}=\left(\mathbf{r}_{p_{1}}+\mathbf{r}_{p_{2}}\right) / 2-\mathbf{r}_{c}, \\
\rho^{2} & =\frac{2}{3}\left(r_{c p_{1}}^{2}+r_{c p_{2}}^{2}\right)+\frac{1}{6} r_{p_{1} p_{2}}^{2}=\frac{1}{2} X^{2}+\frac{4}{3} Y^{2}, \\
\Omega_{\rho} & =\left\{\theta_{\rho}, \Omega_{x}, \Omega_{y}\right\}, \quad \theta_{\rho}=\arctan \left[\sqrt{\frac{3}{8}} \frac{X}{Y}\right] .
\end{aligned}
$$

These Jacobi variables are given in the " $\mathrm{T}$ " Jacobi system (see Fig. 2). The hyperradial components $\chi_{K \gamma}^{(+)}(\rho)$ of the WF in Eq. (2), possessing the pure outgoing asymptotic form

$$
\Psi_{E}^{J M(+)}\left(\rho, \Omega_{\rho}\right)=\rho^{-5 / 2} \sum_{K \gamma}^{K_{\max }} \chi_{K \gamma}^{(+)}(\varkappa \rho) \mathcal{J}_{K \gamma}^{J M}\left(\Omega_{\rho}\right),
$$
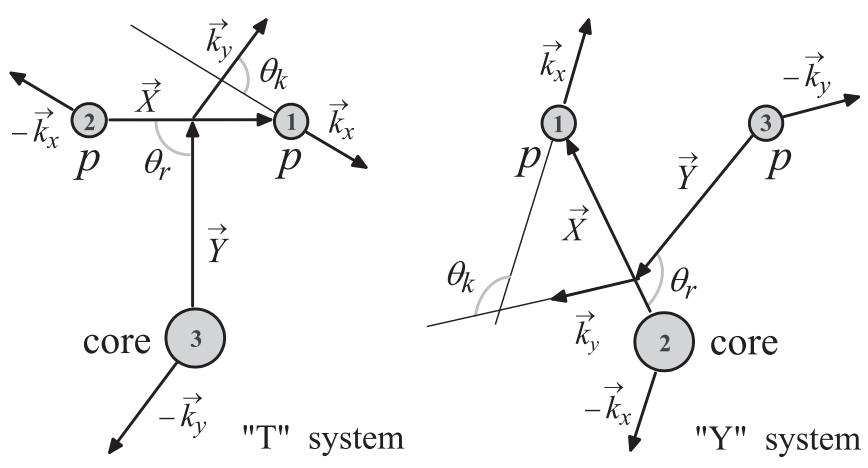

FIG. 2. Independent "T" and "Y" Jacobi systems for the core + $N+N$ three-body system in coordinate and momentum spaces.

are matched to approximate boundary conditions of the threebody Coulomb problem obtained in Ref. [18]. The radial components of this WF at large $\rho$ values can be represented as

$$
\chi_{K \gamma}^{(+)}(\varkappa \rho) \sim A_{K L l_{x} l_{y}}^{J S}(\varkappa) \tilde{\mathcal{H}}_{K \gamma}^{(+)}(\varkappa \rho) .
$$

In general, the functions $\tilde{\mathcal{H}}_{K \gamma}^{(+)}$are some linear combinations of Coulomb functions with the outgoing asymptotic form $G+$ $i F$. The functions $\mathcal{J}_{K \gamma}^{J M}\left(\Omega_{\rho}\right)$ are hyperspherical harmonics (with total orbital momentum $L$ ) coupled with spin functions of nucleons (with spin $S$ ) forming total angular momentum $J$. The "multi-index" $\gamma$ denotes the complete set of quantum numbers except for the principal quantum number $K: \gamma=$ $\left\{L, S, l_{x}, l_{y}\right\}$. The value $K_{\max }$ truncates the hyperspherical expansion. The value $\varkappa$ (hypermoment) is expressed via the energies of the subsystems $E_{x}$ and $E_{y}$ or via the Jacobi momenta $k_{x}$ and $k_{y}$ conjugate to Jacobi coordinates $X$ and $Y$ :

$$
\begin{aligned}
\mathbf{k}_{x} & =\frac{1}{2}\left(\mathbf{k}_{p_{1}}-\mathbf{k}_{p_{2}}\right), \\
\mathbf{k}_{y} & =\frac{2}{3}\left(\mathbf{k}_{p_{1}}+\mathbf{k}_{p_{2}}\right)-\frac{1}{3} \mathbf{k}_{c}, \\
\varkappa^{2} & =2 M E_{T}=2 M\left(E_{x}+E_{y}\right)=2 k_{x}^{2}+\frac{3}{4} k_{y}^{2}, \\
\Omega_{\varkappa} & =\left\{\theta_{\varkappa}, \Omega_{k_{x}}, \Omega_{k_{y}}\right\}, \quad \theta_{\varkappa}=\arctan \left[\sqrt{E_{x} / E_{y}}\right] .
\end{aligned}
$$

A more detailed picture of the "T" and "Y" Jacobi systems in coordinate and momentum spaces can be found in Fig. 2.

The set of coupled equations for the functions $\chi^{(+)}$has the form

$$
\begin{aligned}
& {\left[\frac{d^{2}}{d \rho^{2}}-\frac{\mathcal{L}(\mathcal{L}+1)}{\rho^{2}}+2 M\left\{E-V_{K \gamma, K \gamma}(\rho)\right\}\right] \chi_{K \gamma}^{(+)}(\rho)} \\
& =2 M \sum_{K^{\prime} \gamma^{\prime} \neq K \gamma} V_{K \gamma, K^{\prime} \gamma^{\prime}}(\rho) \chi_{K^{\prime} \gamma^{\prime}}^{(+)}(\rho)+i \Gamma M \chi_{K \gamma}(\rho),
\end{aligned}
$$

where $\mathcal{L}=K+3 / 2$ is the "effective angular momentum" and $V_{K \gamma, K^{\prime} \gamma^{\prime}}(\rho)$ are "three-body potentials" (matrix elements of the pairwise potentials);

$$
V_{K \gamma, K^{\prime} \gamma^{\prime}}(\rho)=\int d \Omega_{\rho} \mathcal{J}_{K^{\prime} \gamma^{\prime}}^{J M *}\left(\Omega_{\rho}\right) \sum_{i<j} V_{i j}\left(\mathbf{r}_{i j}\right) \mathcal{J}_{K \gamma}^{J M}\left(\Omega_{\rho}\right)
$$

and

$$
\Psi_{\text {box }}\left(\rho, \Omega_{\rho}\right)=\rho^{-5 / 2} \sum_{K \gamma} \chi_{K \gamma}(\rho) \mathcal{J}_{K \gamma}^{J M}\left(\Omega_{\rho}\right) .
$$




\section{B. Width and momentum distribution}

Equation (2) is first solved with an arbitrary value of $\Gamma$ and then the width is found according to the "natural" definition as the flux $j$ through a hypersphere with large radius $\rho_{\max }$ divided by the internal normalization $N$ ("number of particles" inside the sphere):

$$
\begin{aligned}
\Gamma_{\text {nat }} & =j / N, \\
j & =\int d \Omega_{\rho} \frac{d j\left(\rho_{\max }, \Omega_{\rho}\right)}{d \Omega_{\rho}}, \\
N & =\sum_{K \gamma} N_{K \gamma}=\sum_{K \gamma} \int_{0}^{\rho_{\text {int }}} d \rho\left|\chi_{K \gamma}^{(+)}(\rho)\right|^{2} .
\end{aligned}
$$

Equation (11) can be derived from the Schrödinger equation in the complex-energy domain using Green's theorem. This theorem requires that $\rho_{\text {int }} \equiv \rho_{\max }$. However, for narrow states (like the situation of radioactive decay) we can in practice limit the integration range by some typical top-of-the-barrier radius $\left(\rho_{\text {int }} \sim 10-20 \mathrm{fm}\right)$ without introducing any uncertainties. What is a reasonable choice of the $\rho_{\text {int }}$ parameter for such comparatively broad systems as ${ }^{6} \mathrm{Be}$ appears to be a question deserving special consideration (see Secs. II D and III A).

The differential flux through the hypersphere at $\rho_{\max }$ is defined as

$$
\frac{d j\left(\rho_{\max }, \Omega_{\rho}\right)}{d \Omega_{\rho}}=\left.\frac{1}{M} \operatorname{Im}\left[\Psi_{3}^{(+) \dagger} \rho^{5 / 2} \frac{d}{d \rho} \rho^{5 / 2} \Psi_{3}^{(+)}\right]\right|_{\rho=\rho_{\max }}
$$

If, for sufficiently large $\rho_{\max }$, the coefficients $A_{L l_{x} l_{y}}^{K S}$ in Eq. (6) become independent of $\rho$, then the coordinate distribution becomes identical to the momentum distribution, i.e.,

$$
\frac{d j\left(\rho_{\max }, \Omega_{\rho}\right)}{d \Omega_{\rho}} \rightarrow \frac{d j\left(\Omega_{\varkappa}\right)}{d \Omega_{\varkappa}} .
$$

Further discussions of the validity of this approximation [Eq. (15)], and detailed expressions for the momentum distributions, can be found in Ref. [18].

\section{Potentials}

The Coulomb potential of the homogeneously charged sphere of radius $r_{\mathrm{sph}}=1.131$ and $1.852 \mathrm{fm}$ is used in the $p-p$ and $\alpha-p$ channels, respectively.

The nuclear $N N$ potential is taken either as a simple $s$-wave single-Gaussian form BJ (from the book of Brown and Jackson [25])

$$
V(r)=V_{0} \exp \left(-r^{2} / r_{0}^{2}\right),
$$

with $V_{0}=-31 \mathrm{MeV}$ and $r_{0}=1.8 \mathrm{fm}$ or the quasirealistic "soft-core" potential GPT (Gogny-Pires-de Tourreil [26]).

In the core- $p$ channel, we use an $\ell$-dependent potential SBB (Sack-Biedenharn-Breit [27])

$$
V(r)=V_{c}^{(\ell)} \exp \left(-r^{2} / r_{0}^{2}\right)+(\ell \cdot \mathbf{s}) V_{\ell s} \exp \left(-r^{2} / r_{0}^{2}\right),
$$

where $r_{0}=2.30 \mathrm{fm}, V_{c}^{(0)}=50 \mathrm{MeV}, V_{c}^{(1)}=-47.32 \mathrm{MeV}$, $V_{c}^{(2)}=-23 \mathrm{MeV}$, and $V_{l s}=-11.71 \mathrm{MeV}$. Historically, a somewhat modified SBBM potential has been used in the calculations of $A=6$ isobars to better reproduce the binding energies (e.g., Ref. [28]). Later it was realized that it is more consistent to provide the phenomenological binding-energy correction using an additional short-range three-body potential (see, e.g., the discussion in Ref. [23]). In this work, we used a short-range three-body potential of the form

$$
V_{3}(\rho)=\delta_{K \gamma, K^{\prime} \gamma^{\prime}} V_{3}^{(0)} /\left[1+\exp \left(\left(\rho-\rho_{0}\right) / d_{3}\right)\right]
$$

where $\rho_{0}=2.5 \mathrm{fm}$ and $d_{3}=0.4 \mathrm{fm}$. This "short-range" threebody potential (note the small diffuseness) does not distort the interactions in the sub-barrier region that was found to be important for consistent studies of the decay properties.

Three sets of nuclear potentials are employed in this work. They are denoted as P1 (SBB+BJ), P2 (SBB+GPT), and P3 $(\mathrm{SBBM}+\mathrm{GPT})$. The values of $V_{3}^{(0)}$ used with the potential sets P1, P2, and P3 are $-11.14,-13.22$, and $-0.64 \mathrm{MeV}$, respectively. Throughout this article when the potential set is not specified, the results of the calculations with the P2 set are shown.

\section{Model for charge-exchange reaction}

In general, different definitions for the width of a decaying state coincide only in the limit when the width is very small. For the ground state of ${ }^{6} \mathrm{Be}$, the definition of Eq. (11) is not very precise, as this state is comparatively broad $(\Gamma=92 \pm$ $6 \mathrm{keV}$ [15]) and thus the internal normalization $N$ [Eq. (13)] is sensitive to the integration limit $\rho_{\text {int }}$. For reasonable values of $\rho_{\text {int }}$ ranging from 10 to $20 \mathrm{fm}$, the uncertainty in the width $\Gamma_{\text {nat }}$ is about $25 \%$ [see Sec. III C, Fig. 7(a)]. This problem does not exist for narrow $2 p$ emitters $(\Gamma<1 \mathrm{eV})$ where the WFs $\chi_{K \gamma}^{(+)}$are vanishingly small as they tunnel under the Coulomb barrier. The densities for the dominant components of the ${ }^{6} \mathrm{He}$ and ${ }^{6} \mathrm{Be}$ WFs are shown in Fig. 3. For ${ }^{6} \mathrm{Be}$, it is clear that the WFs under the barrier are not negligible.

For sufficiently broad states, there are alternative ways to derive the width. These involve either the study of the $3 \rightarrow 3$ scattering or the study of a particular reaction. For technical reasons, the latter is preferable for our application. For example, to determine the population of ${ }^{6} \mathrm{Be}$ in a chargeexchange reaction on ${ }^{6} \mathrm{Li}$ at zero angle, Eq. (2) can be

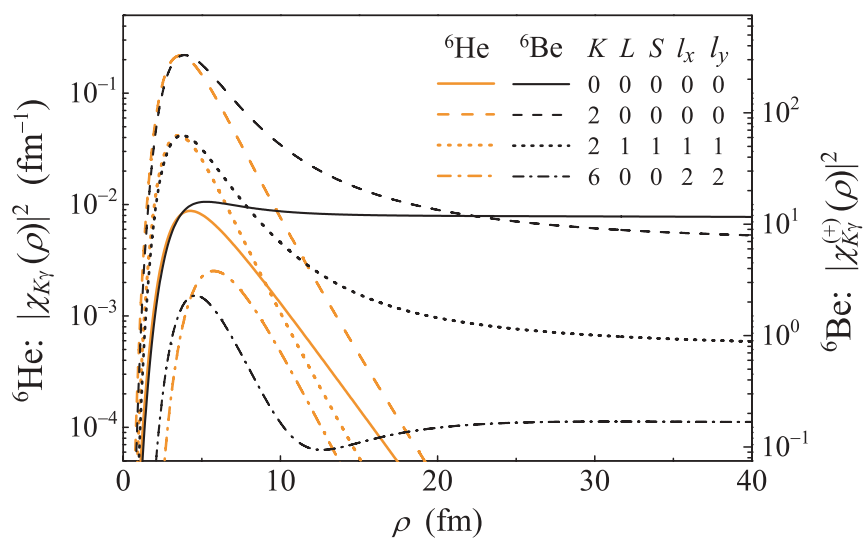

FIG. 3. (Color online) Densities $\left|\chi_{K \gamma}(\rho)\right|^{2}$ and $\left|\chi_{K \gamma}^{(+)}(\rho)\right|^{2}$ for the largest components of the ${ }^{6} \mathrm{He}$ and ${ }^{6} \mathrm{Be}$ g.s. WFs. 
reformulated as

$$
\left(\hat{H}-E_{T}\right) \Psi_{{ }^{\mathrm{Be}}}^{(+)}\left(\rho, \Omega_{\rho}\right)=\sum_{i} \tau_{i}^{-} \sum_{M} \sigma_{i}^{(M)} \Psi_{{ }_{6}^{\mathrm{Li}}}^{J M}\left(\rho, \Omega_{\rho}\right) .
$$

This notation is based on the fact that for angles close to zero, the transitions in charge-exchange reactions, in the limit of high energies, are provided by the Gamow-Teller operator.

Using the source function of Eq. (19), the cross section for the population of the three-body continuum is proportional to the outgoing flux of the three particles on a hypersphere of some large radius $\rho=\rho_{\max }$ :

$$
d \sigma\left(E_{T}\right) / d E_{T} \sim j\left(\rho_{\max }, \Omega_{\rho}\right)
$$

Differentials of this flux on the hypersphere provide angular and energy distributions among the decay products at the given decay energy $E_{T}$ in analogy with Eqs. (14) and (15).

For the derivation of the ${ }^{6} \mathrm{Be}$ decay properties, we have the general decay method of Eq. (11) which, however, is sensitive to an ill-defined parameter. We also have introduced the method of Eq. (20), which is relevant to certain reactions but not to the one considered in the experimental part of this work. The use of both of these methods in this work may be confusing so it is important that we clearly outline our logic in using them.

(i) For ${ }^{6} \mathrm{Be}$, the general decay approach of Eq. (11) has insufficient precision because the result is sensitive to the parameter $\rho_{\text {int }}$ when it is varied by tens of percentages. This means that we need a more specific formulation for the decay properties incorporating a particular reaction mechanism.

(ii) Charge-exchange reactions on ${ }^{6} \mathrm{Li}$ allow the very simple theoretical formulation given in Eq. (19) when restricted to particular kinematical conditions.

(iii) It is possible to demonstrate that the decay characteristics of the ${ }^{6} \mathrm{Be}$ g.s. are stable with respect to the particular choice of the right-hand side of Eq. (19) and thus to the choice of reaction. Therefore Eq. (19) can also be used when ${ }^{6} \mathrm{Be}$ is created in other reactions.

(iv) The parameter $\rho_{\text {int }}$ in Eq. (11) can be fine tuned to reproduce the widths obtained with Eq. (20). It can be checked that this fine tuning should be done once and then the definitions of Eqs. (11) and (20) provide equivalent results for calculations performed with different model parameters.

(v) In this work we used both the approaches of Eqs. (11) and (20). Equation (20) allows us to obtain energy profiles of the g.s. population cross section while Eq. (11) is effective as an "economic" method when massive calculations with different model parameters are performed. In this case, the WF needs only to be calculated once (on resonance), whereas calculations are needed for 10-20 points when Eq. (20) is used.

We should emphasize that the validity of the statements given in items (iii) and (iv) is specific for the ${ }^{6} \mathrm{Be}$ g.s. This was not known in advance and was checked in this work.

\section{E. "Feshbach" reduction}

Although the $\mathrm{HH}$ calculations for ${ }^{6} \mathrm{Be}$ can be performed with $K_{\max }=22-26$, these basis sizes may not be sufficient to obtain good convergence for all observables. However, the basis size can be effectively increased using the adiabatic procedure based on the so-called Feshbach reduction (FR) [23]. Feshbach reduction eliminates from the total WF, $\Psi=$ $\Psi_{p}+\Psi_{q}$, an arbitrary subspace $q$ using the Green's function of this subspace:

$$
H_{p}=T_{p}+V_{p}-V_{p q} G_{q} V_{p q} .
$$

In an adiabatic approximation, we can assume that the radial part of the kinetic energy is small under the centrifugal barrier in the channels where this barrier is large and can be approximated as a constant. In this approximation, the FR procedure is reduced to the construction of effective three-body interactions $V_{K \gamma, K^{\prime} \gamma^{\prime}}^{\text {eff }}$ by the matrix operations

$$
V_{K \gamma, K^{\prime} \gamma^{\prime}}^{\mathrm{eff}}=V_{K \gamma, K^{\prime} \gamma^{\prime}}-\sum V_{K \gamma, \bar{K} \bar{\gamma}} G_{\bar{K} \bar{\gamma}, \bar{K}^{\prime} \bar{\gamma}^{\prime}} V_{\bar{K}^{\prime} \bar{\gamma}^{\prime}, K^{\prime} \gamma^{\prime}},
$$

where the approximate Green's function is given by

$$
\begin{aligned}
G_{K \gamma, K^{\prime} \gamma^{\prime}}^{-1}= & \left(H-E_{T}\right)_{K \gamma, K^{\prime} \gamma^{\prime}}=V_{K \gamma, K^{\prime} \gamma^{\prime}} \\
& +\left[E_{f}-E_{T}+\frac{(K+3 / 2)(K+5 / 2)}{2 M \rho^{2}}\right] \delta_{K \gamma, K^{\prime} \gamma^{\prime}} .
\end{aligned}
$$

Summations over the indices with the bar are made for the eliminated channels. We typically eliminate the channels with $K>K_{F R}$, where $K_{F R}$ provides the sector of the hyperspherical basis where the calculations remains fully dynamical. We take the "Feshbach energy" $E_{f}$ in our calculations as $E_{f} \equiv E_{T}$.

There are two ways to control the reliability of the FR procedure. (i) The "soft" method is to vary $K_{F R}$ from the maximum attainable in the dynamic calculations downward for fixed $K_{\max }$. The results, in principle, should coincide. (ii) The "safe" method is to take $K_{\max }$ in the range attainable for dynamic calculations and compare the "reduced" $K_{\max } \rightarrow K_{F R}$ calculations (with much smaller dynamic basis size $K_{F R}$ ) with completely dynamic calculations with $K_{\max }$. For ${ }^{6} \mathrm{Be}$, these considerations show that we can safely use $K_{F R}=14$. However, the even safer value of $K_{F R}=22$ is used in this work.

\section{RESULTS FOR GROUND STATE}

There are several convergence characteristics that should be understood before reliable results on ${ }^{6} \mathrm{Be}$ can be obtained. The convergence character is quite different for each of the observables of interest and it also depends strongly on the interaction in the $p$ - $p$ channel.

\section{A. Convergence of energy and width}

Because of the problem mentioned in Sec. II D, we need to begin our studies with the energy dependence of the cross section. The convergence of the cross-section energy profile with increasing size of the basis is demonstrated in Fig. 4. The main character of the convergence is clearly seen here; the 


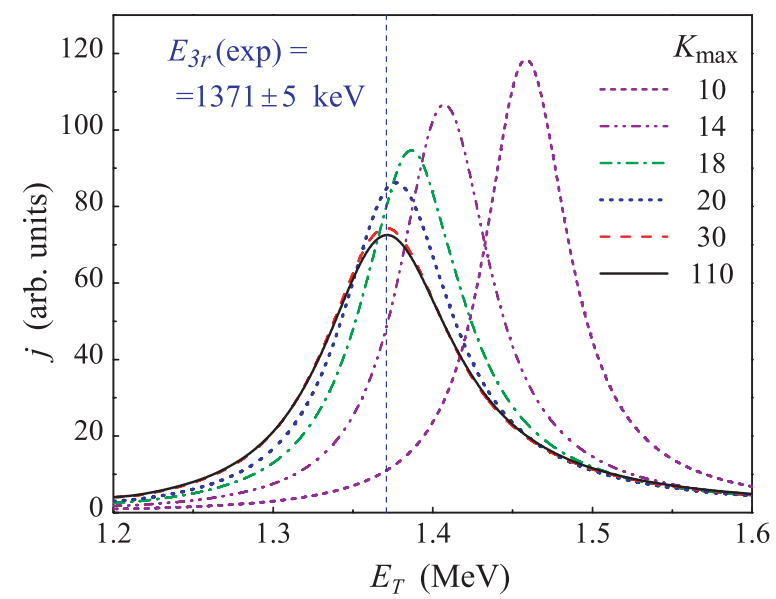

FIG. 4. (Color online) The energy profile of the ${ }^{6} \mathrm{Be}$ g.s. populated in the charge-exchange reaction with ${ }^{6} \mathrm{Li}$. The results are shown as a function of the basis size $K_{\max }$ where $K_{F R}=22$. For $K_{\max } \leqslant K_{F R}$, Feshbach reduction is not needed. Predictions were obtained with the P1 potential set.

centroid energy decreases, while the width grows significantly. The calculations shown in Fig. 4 are practically converged by $K_{\max }=30$ and further increases of the effective basis size up to $K_{\max }=110$ lead to only minor changes in the energy profile.

The cross section for the ${ }^{6} \mathrm{Be}$ g.s. population, shown in Fig. 4, clearly has a profile close to a slightly asymmetric Lorentzian. Can the profile of this three-body resonance be described by an appropriately-modified $R$-matrix-type expression? A curious result is obtained here, the resonance profile, shown in Fig. 4 by the solid curve, can be fit with amazing precision by the following expression:

$$
\begin{aligned}
\sigma\left(E_{T}\right) & \sim \frac{\Gamma\left(E_{T}\right)}{\left(E_{T}-E_{3 r}\right)^{2}+\Gamma\left(E_{T}\right)^{2} / 4}, \\
\Gamma\left(E_{T}\right) & =\Gamma_{0}\left[\alpha\left(\frac{E_{T}}{E_{3 r}}\right)^{2}+(1-\alpha)\left(\frac{E_{T}}{E_{3 r}}\right)^{4}\right],
\end{aligned}
$$

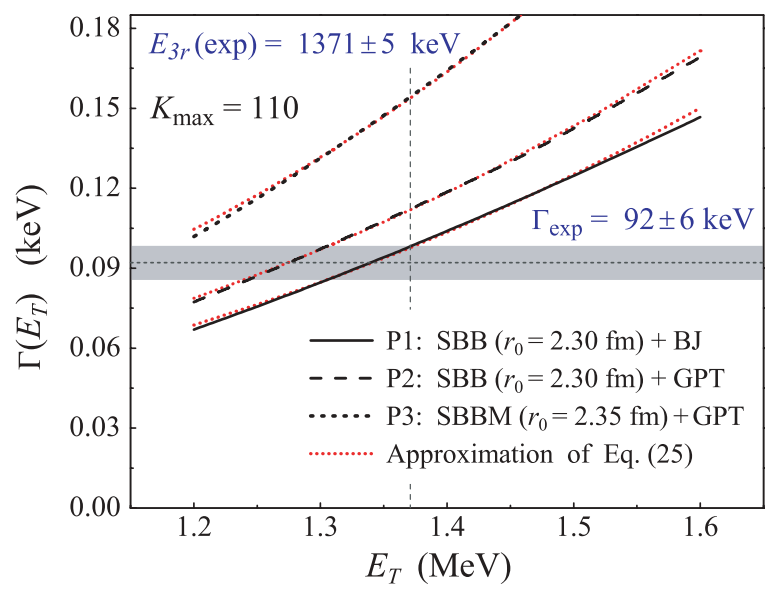

FIG. 5. (Color online) The dependence of the ${ }^{6} \mathrm{Be}$ g.s. width on the decay energy $E_{T}$. Predications are shown for the three potential sets P1-P3. The dotted curves show the approximation of Eq. (25).

where $\Gamma_{0}=98 \mathrm{keV}$ and $\alpha=0.65$. Equation (24) is the ordinary expression for the inelastic cross section of an isolated resonance. The parametrization of Eq. (25) was chosen because, for the single-channel penetration through the hyperspherical barrier with $K=0$, the energy dependence of the width can be inferred as $\Gamma\left(E_{T}\right) \sim E_{T}^{2}$. For $K=2$ one has $\Gamma\left(E_{T}\right) \sim E_{T}^{4}$ (see, e.g., Ref. [29]). It should be understood that the $K=0$ component is equivalent to a "phase volume" with the characteristic energy behavior of $\sim E_{T}^{2}$. The energy dependence of the width obtained by Eq. (25) almost coincides with the calculated dependence of this width in a reasonable energy range, see Fig. 5. This figure shows also the comparison for potentials $\mathrm{P} 2$ and $\mathrm{P} 3$ [one uses $\alpha=0.63$ and 0.52 in Eq. (25), respectively]. If we take the actual calculated partial widths for the $K=0$ and 2 components from Table I, then the value of $\alpha$ can be estimated as

$$
\alpha=\Gamma_{K=0} /\left(\Gamma_{K=0}+\Gamma_{K=2}\right) \approx 0.58
$$

TABLE I. Weights $N_{i}$ of the dominating components of the ${ }^{6} \mathrm{He}$ and ${ }^{6} \mathrm{Be}$ g.s. WFs and the fractional partial widths of the ${ }^{6} \mathrm{Be}$ g.s. WF

\begin{tabular}{|c|c|c|c|c|c|c|c|c|c|c|c|c|c|c|}
\hline \multicolumn{6}{|c|}{ Quantum numbers } & \multicolumn{3}{|c|}{$N_{i}\left({ }^{6} \mathrm{He}\right)$} & \multicolumn{3}{|c|}{$N_{i}\left({ }^{6} \mathrm{Be}\right)$} & \multicolumn{3}{|c|}{$\Gamma_{i} / \Gamma\left({ }^{6} \mathrm{Be}\right)$} \\
\hline$i$ & $K$ & $L$ & $S$ & $l_{x}$ & $l_{y}$ & $\mathrm{P} 1$ & $\mathrm{P} 2$ & P3 & $\mathrm{P} 1$ & P2 & P3 & P1 & $\mathrm{P} 2$ & P3 \\
\hline 1 & 0 & 0 & 0 & 0 & 0 & 4.32 & 4.65 & 4.27 & 6.72 & 7.24 & 6.65 & 50.44 & 50.77 & 41.03 \\
\hline 2 & 2 & 0 & 0 & 0 & 0 & 78.36 & 80.73 & 79.40 & 75.71 & 77.49 & 75.28 & 33.48 & 33.74 & 41.52 \\
\hline 3 & 2 & 1 & 1 & 1 & 1 & 14.19 & 11.28 & 12.02 & 13.09 & 10.60 & 11.44 & 3.89 & 3.31 & 6.15 \\
\hline 4 & 4 & 0 & 0 & 0 & 0 & 0.03 & 0.04 & 0.02 & 0.10 & 0.14 & 0.07 & 2.03 & 2.11 & 2.25 \\
\hline 5 & 4 & 0 & 0 & 2 & 2 & 0.48 & 0.50 & 0.58 & 0.44 & 0.45 & 0.53 & 6.10 & 6.48 & 4.97 \\
\hline 6 & 6 & 0 & 0 & 0 & 0 & 0.01 & 0.02 & 0.01 & 0.02 & 0.03 & 0.01 & 1.63 & 1.26 & 1.49 \\
\hline 7 & 6 & 0 & 0 & 2 & 2 & 1.13 & 1.18 & 1.56 & 1.56 & 1.60 & 2.32 & 0.67 & 0.73 & 0.78 \\
\hline 8 & 6 & 1 & 1 & 3 & 3 & 0.57 & 0.54 & 0.75 & 0.79 & 0.75 & 1.18 & 0.08 & 0.06 & 0.09 \\
\hline 9 & 8 & 0 & 0 & 0 & 0 & 0.28 & 0.31 & 0.37 & 0.47 & 0.51 & 0.66 & 0.85 & 0.69 & 0.85 \\
\hline 10 & 8 & 0 & 0 & 2 & 2 & 0.17 & 0.17 & 0.25 & 0.28 & 0.28 & 0.46 & 0.08 & 0.11 & 0.10 \\
\hline 11 & 8 & 0 & 0 & 4 & 4 & 0.03 & 0.03 & 0.04 & 0.05 & 0.05 & 0.08 & 0.37 & 0.40 & 0.32 \\
\hline
\end{tabular}
in percentages. The results are for the Jacobi " $\mathrm{T}$ " system. The normalizations of the ${ }^{6} \mathrm{Be}$ components are found for the integration radius $\rho_{\text {int }}=12.5 \mathrm{fm}$. 
This is quite close to the value 0.65 obtained by a fit. This is true also for potentials $\mathrm{P} 2$ and $\mathrm{P} 3$, for which corresponding values are 0.58 and 0.46 .

The existence of this simple approximation, despite the fact that there are Coulomb interactions and other numerous channels involved, may demonstrate that the dynamics of the ${ }^{6} \mathrm{Be}$ g.s. decay is largely defined by the penetration through the hyperspherical barriers. Possibly, this is due to the comparatively large ${ }^{6} \mathrm{Be}$ decay energy of $E_{3 r}=1.371 \mathrm{MeV}$. Simple estimates show that this state has an energy close to the value at the top of the Coulomb barrier.

It was found that the value of $j\left(E_{T}\right)$ for the ${ }^{6} \mathrm{Be}$ g.s. is not sensitive to the particular choice of the source in Eq. (19). For strong variations of the source term (e.g., $\pm 50 \%$ in size or arbitrary change of the relative weights of the components), typical variations obtained from Fig. 4 are within the width of the line and there are no noticeable differences in the calculated momentum distributions (there are still some exclusive situations, which we will discuss elsewhere). This means that for observable properties of the ${ }^{6} \mathrm{Be}$ g.s. there is no sensitivity to the reaction mechanism and the width defined by the procedure of Eqs. (24) and (25) is very reliable. This absence of sensitivity to the reaction mechanism also means that the general decay definition of Eq. (11) should provide stable results and the difficulty connected with the poorly defined parameter $\rho_{\text {int }}$ can be overcome. We can fine tune the value $\rho_{\text {int }}$ in Eq. (13) so that the definition of the width in Eq. (11) coincides with the definition in Eq. (24) and check that the equivalence of the definitions does not depend on energy and other model settings. We found that all the potential sets P1-P3 need $\rho_{\text {int }} \approx 12.5 \mathrm{fm}$. Subsequently, we can reliably use Eq. (11).

The convergences of the predicted resonance energy and width as a function of the hyperspherical basis size are shown in Fig. 6 for each of the potential sets. In all cases, our calculations have fully converged. The resonance energies are forced to approach the experimental value $E_{3 r}=1.371 \mathrm{MeV}$. This is achieved by fine tuning the phenomenological potential of Eq. (18). Keeping the calculated decay energy identical

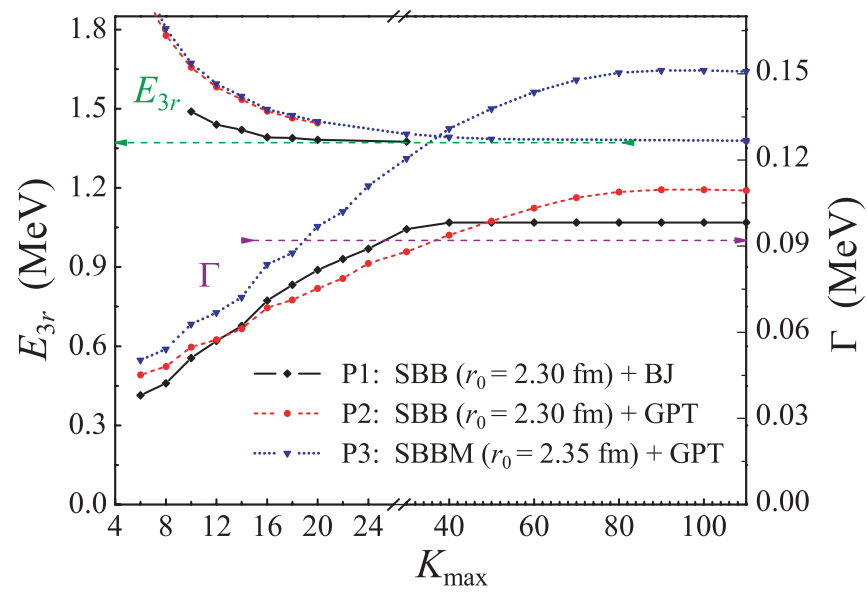

FIG. 6. (Color online) The convergency of the resonance energy $E_{3 r}$ and the width $\Gamma$ of the ${ }^{6} \mathrm{Be}$ g.s. as a function of the basis size $K_{\max }$. Note the change of the scale at the horizontal axis.
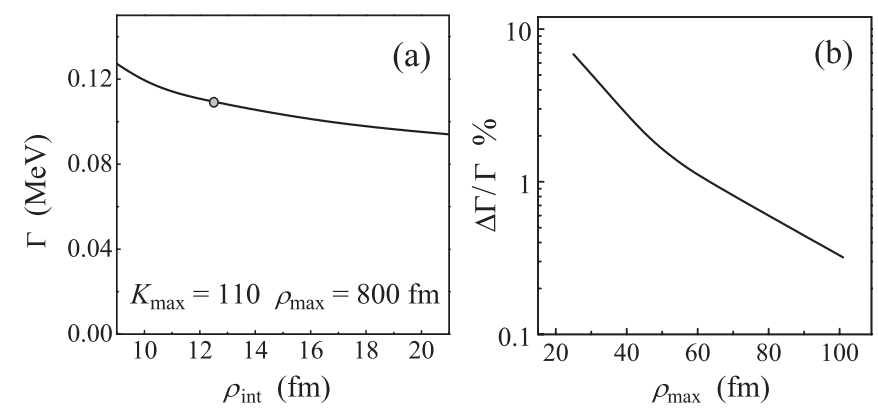

FIG. 7. (a) The sensitivity of the width as defined in Eq. (11) to the size of the "internal region" $\rho_{\text {int }}$. The circle shows the value of $\rho_{\text {int }}$ at which this width coincides with that defined via the cross-section profile in Eqs. (19), (20), (24), and (25). (b) Relative precision of the width as a function of the matching radius $\rho_{\max }$.

to the experimental value is necessary to provide reasonable predictions for the rest of the decay characteristics. We can see that while the calculations with P1 and P2 (SBB potential in the $\alpha-p$ channel) are in good agreement with each other and with the experimental value, the width obtained with P3 (SBBM potential) is far too large.

An expected feature observed here is the much slower convergence of the calculations with a quasirealistic potential in the $N N$ channel. An important, but often disregarded, fact, which one can see in Fig. 6, is the much slower convergence of the width as compared to the energy. This means that, in general, an energy convergence does not guarantee the convergence of other important characteristics. As we will see in Sec. III C, the situation with momentum distributions is even more complicated than it is for the widths.

The sensitivity of the width to a number of the other parameters in the calculations is demonstrated in Fig. 7. Figure 7(a) shows the sensitivity of the width defined by Eq. (11) to the size $\rho_{\text {int }}$ of the region where the internal normalization is calculated. The stability of the calculations to the dynamical range $\rho_{\max }$ is demonstrated in Fig. 7(b). To attain $1 \%$ numerical precision in the width calculations, one needs to go beyond $60 \mathrm{fm}$ in the hyperradius $\rho$.

\section{B. Features of the momentum distributions from ${ }^{6} \mathrm{Be}$ decay}

The correlations in the decay of ${ }^{6} \mathrm{Be}$ include both the generic features of the $2 p$ decays, as discussed earlier in Refs. [14,18,22,23], and some peculiarities that we present in more detail now. For nuclear states with $J \leqslant 1 / 2$ (as is the case for the ${ }^{6} \mathrm{Be}$ g.s. decay), the three-body momentum correlations (averaged over the final-state spins) can be completely described by two parameters. In total, there are nine degrees of freedom for three particles in the final state. Of these, three describe the center-of-mass motion, three describe the Euler rotation of the decay plane (for $J \leqslant 1 / 2$ all its orientations are quantum-mechanically identical), and the three-body decay energy is fixed. Thus we are left with two parameters to describe the correlations. It is convenient to choose the energy distribution parameter $\varepsilon$ between any two 


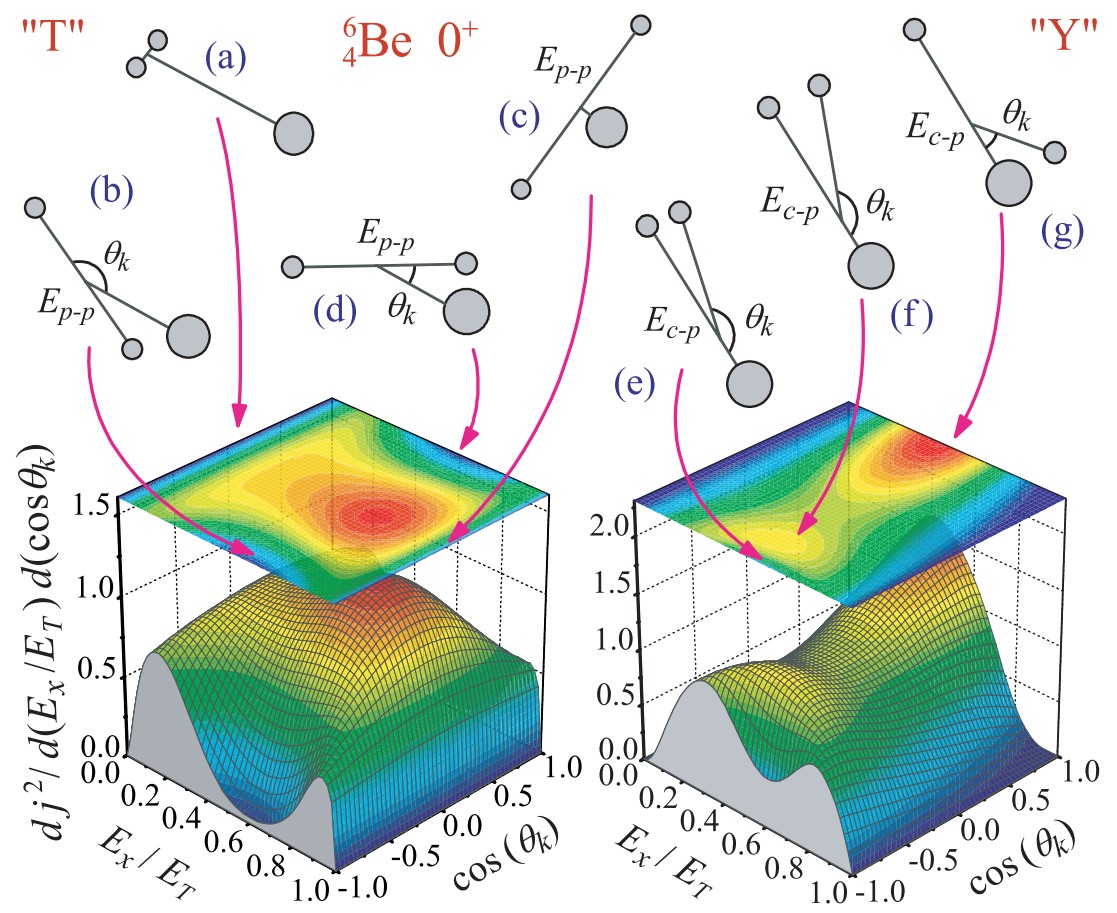

FIG. 8. (Color online) Complete correlation picture for the ${ }^{6} \mathrm{Be}$-g.s. decay, presented in the " $T$ " and "Y" Jacobi systems. Schematic diagrams (a-g) indicate the correlations associated with selected regions of these plots. of the particles and the angle $\theta_{k}$ between the Jacobi momenta:

$$
\varepsilon=E_{x} / E_{T}, \quad \cos \left(\theta_{k}\right)=\frac{\mathbf{k}_{x} \cdot \mathbf{k}_{y}}{k_{x} k_{y}} .
$$

These parameters can be constructed in any Jacobi system and for ${ }^{6} \mathrm{Be}$ there are two "irreducible" Jacobi systems, called "T" and "Y", see Fig. 2. The distributions constructed in different Jacobi systems are just different representations of the same physical picture. However, different aspects of the correlations may be better revealed in a particular Jacobi system.

Predictions for the complete correlation picture of ${ }^{6} \mathrm{Be}$ g.s. decay are shown in Fig. 8 for both the "T" and "Y" Jacobi systems. Schematic diagrams are included in this figure to help in visualizing the correlations associated with different regions of the Jacobi plots. The main features of these distributions are:

(i) The energy distribution in the "T" system has a doublehumped profile that is an indication of the $\left[p^{2}\right]$ configuration (two protons in the $p$ shell) dominance that was pointed out in very early articles on ${ }^{6} \mathrm{Be}$ [13,30-32]. This double-humped configuration is expressed more in coordinate space (the internal region of the ${ }^{6} \mathrm{He}$ and ${ }^{6} \mathrm{Be}$ WFs can be seen in Fig. 9). However in the asymptotic region, the double-humped structure only marginally "survives." The internal peaks in Fig. 9 have the special names of "diproton" (protons are close to each other) and "cigar" (protons are in-line with $\alpha$-particle) configurations [28].

(ii) There are kinematic regions where the presence of events is suppressed due to Coulomb repulsions. Strong
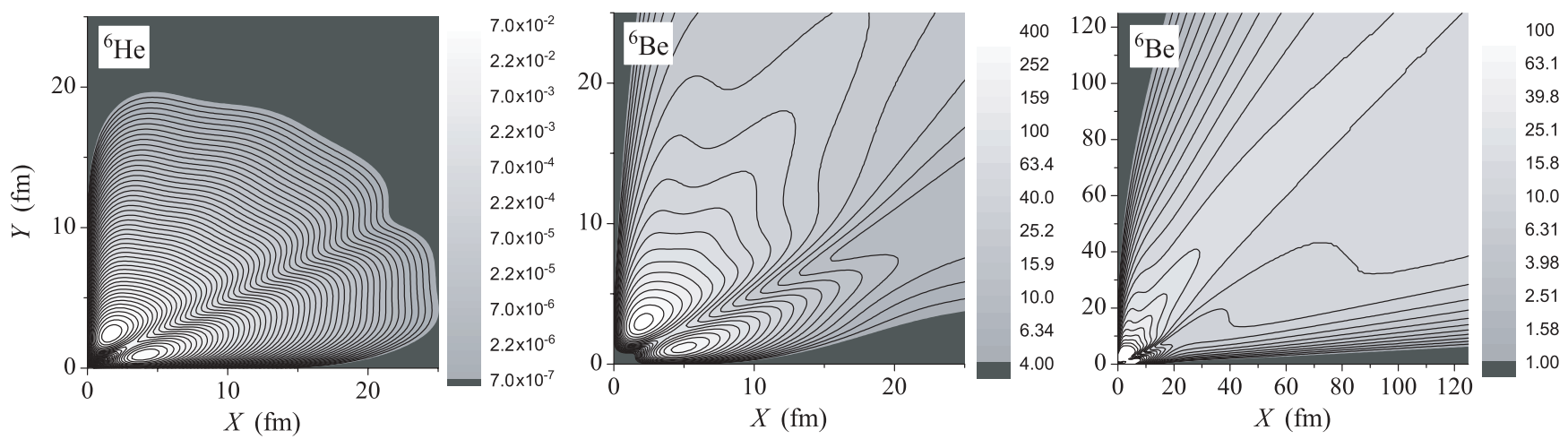

FIG. 9. Spatial correlation densities $|\Psi(X, Y)|^{2}$ for the ${ }^{6} \mathrm{He}$ and ${ }^{6} \mathrm{Be}$ g.s. WFs in the "T" system. For ${ }^{6} \mathrm{Be}$, two panels provide the view in different radial ranges. Pay attention to difference in the scales. The variation from the top to the thick contour line in the ${ }^{6} \mathrm{He}$ panel corresponds to the whole scale variation in the two ${ }^{6} \mathrm{Be}$ panels. 

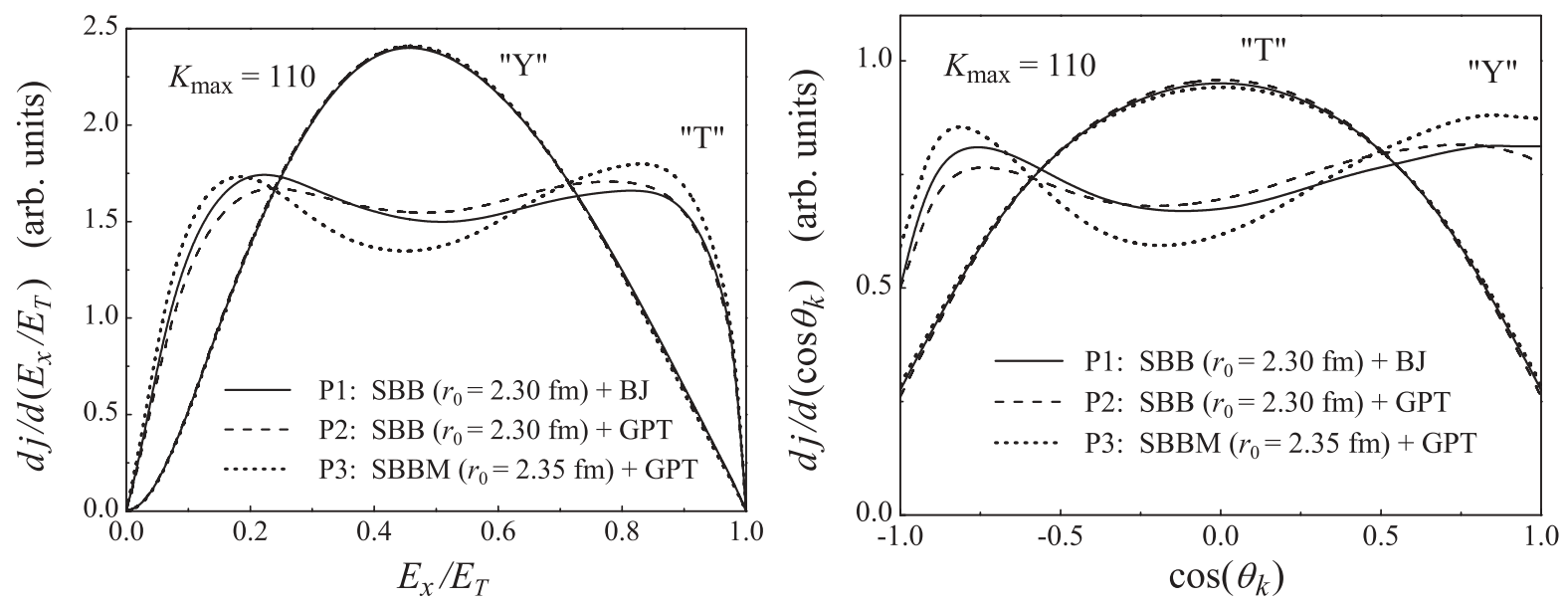

FIG. 10. Sensitivity of the energy and angular distributions in the decay of the ${ }^{6}$ Be g.s. to the choice of the potential set. Results are shown for both the "T" and "Y" Jacobi systems.

suppressions are predicted in the $\alpha-p$ channel in regions labeled by the schematic diagrams (b) and (d) in Fig. 8. A smaller suppression in the $p$ - $p$ channel in region (e) is also predicted.

(iii) There are enhancements due to the $p$ - $p$ final-state interaction in regions (a) and (f). The ${ }^{5} \mathrm{Li}$ g.s. resonance in the $\alpha-p$ channel is not accessible for decay. However, some hint of its presence can be obtained from the enhancement in region ( $\mathrm{g})$. This is a "back-to-back" configuration, where protons fly in the opposite directions. However, the reason for the enhancement of such a configuration is not fully understood.

(iv) The angular dependence in the " $T$ " system almost vanishes for regions (a) and (c) $\left(E_{x} / E_{T} \sim 0\right.$ and $\left.E_{x} / E_{T} \sim 1\right)$. It is clear that in the limit $E_{x} / E_{T} \rightarrow 0$ and $E_{x} / E_{T} \rightarrow 1$ the dependence on the relative orientation of $\mathbf{k}_{x}$ and $\mathbf{k}_{y}$ should become degenerate. However, at intermediate values of $E_{x} / E_{T}$, this dependence is very pronounced.

(v) The energy distribution in the " $Y$ " system is almost a symmetric bell shape. This is most clearly seen in the projected distribution of Fig. 10. This energy distribution is between the core and one of the protons and its symmetry reflects the symmetry between protons. In heavy two-proton emitters this distribution becomes very narrow and practically symmetric.

The predicted correlations shown in Fig. 8 are obtained on resonance. The dependence of the energy correlation on the decay energy of ${ }^{6} \mathrm{Be}$ is displayed in Fig. 11. The doublehumped shape of this spectrum becomes less pronounced with decreasing energy. With smaller energy, the relative contribution of the $\left[s^{2}\right]$ configuration to the decay grows compared to the $\left[p^{2}\right]$ configuration. The latter has an additional centrifugal component to the barrier and its contribution to the width should be suppressed at low energies. The pure $\left[s^{2}\right]$ configuration should produce a featureless "phase-volume" energy distribution

$$
d j / d E_{x} \sim \sqrt{E_{x}\left(E_{T}-E_{x}\right)} .
$$

The sensitivity of the projected distributions to the choice of the potential set P1-P3 is demonstrated in Fig. 10. The angular distribution in the " $\mathrm{T}$ " system and the energy distribution in "Y" systems are practically insensitive to this choice. The other projected distributions demonstrate sensitivity on the level of $10-15 \%$. However, local differences in certain kinematic regions are much larger.

Figures 10 and 11 demonstrate what we call the "softness" of the ${ }^{6} \mathrm{Be}$ system: minor variations in the conditions or computational details lead to noticeable variations in the observable properties. Heavier $2 p$ emitters appear to be much "stiffer" in this respect.

\section{Convergence of the momentum distributions}

In our calculations there are two projected distributions which are practically insensitive to convergence issues (the angular distribution in the " $\mathrm{T}$ " system and the energy distribution

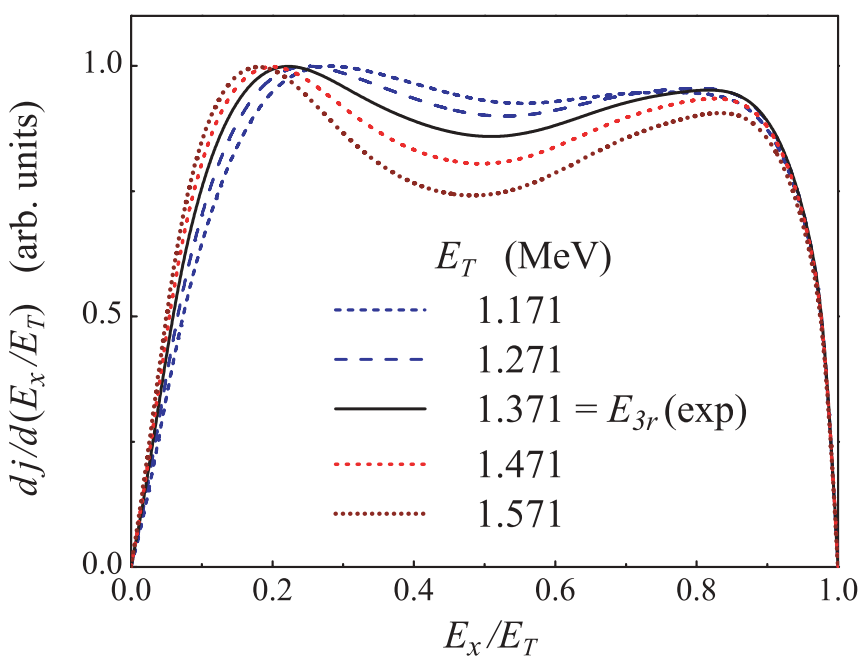

FIG. 11. (Color online) The dependence of energy distribution between the proton ("T" system) in the decay of the ${ }^{6} \mathrm{Be}$ g.s. on the decay energy $E_{T}$. 


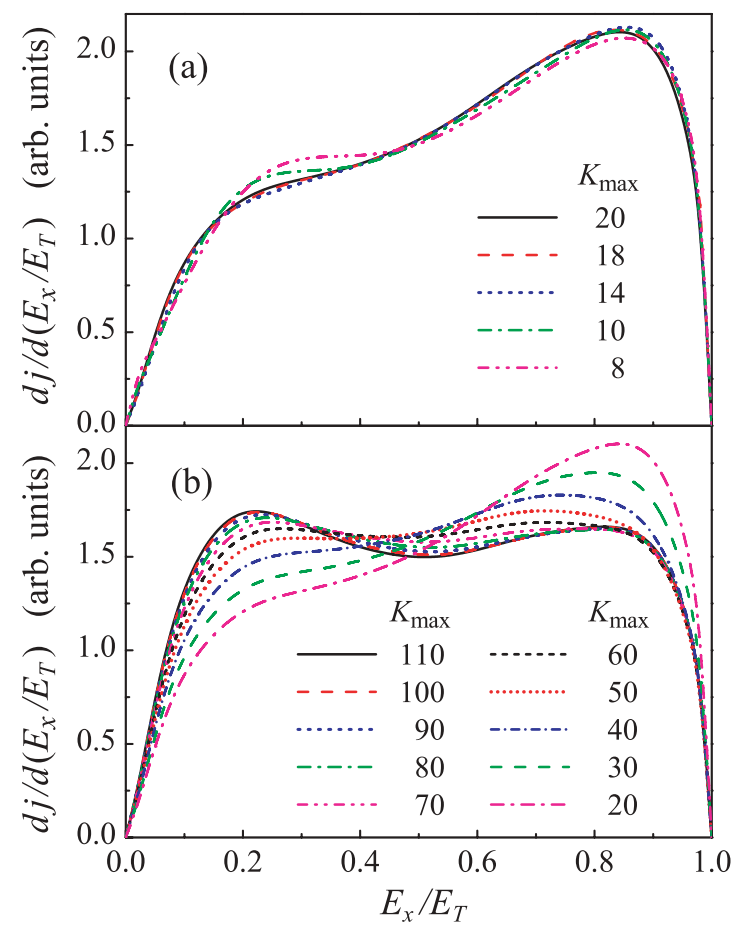

FIG. 12. (Color online) Convergency of the "T" energy distribution in the decay of the ${ }^{6} \mathrm{Be}$ g.s. as a function of the basis size $K_{\max }$.

in the "Y" system). The other two distributions (the angular distribution in the "Y" system and the energy distribution in the " $T$ " system) demonstrate strong sensitivity. The convergence of the energy distributions are illustrated in Figs. 12 and 13.

The convergence of the energy distribution between protons has a very curious character, see Fig. 12 . From $K_{\max }=8$ to $K_{\max }=22$ this distribution is very stable [several curves almost coincide, see Fig. 12(a)]. Then from $K_{\max }=24$ to $K_{\max } \sim 70$, the distribution changes qualitatively, and up to $K_{\max } \sim 100$ there is still a noticeable variation [Fig. 12(b)].

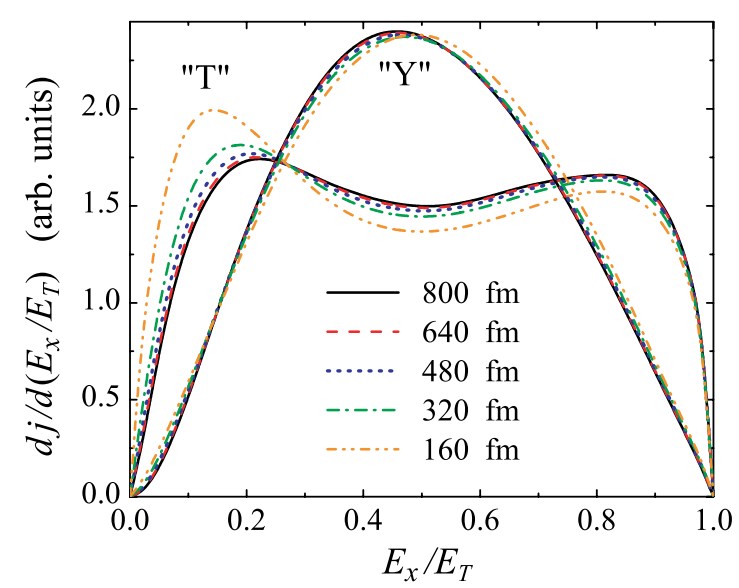

FIG. 13. (Color online) Dependence of energy distribution in the decay of the ${ }^{6} \mathrm{Be}$ g.s. on the maximal dynamic range of the calculation $\rho_{\max }$. For the " $T$ " Jacobi system, $E_{x}$ is the energy between two protons, and in the "Y" Jacobi system, $E_{x}$ is the energy between core and one of the protons.
Hopefully with $K_{\max }=110$, we have a well converged distribution. Calculations with small basis sizes (e.g., $K_{\max } \leqslant 70$ ) for ${ }^{6} \mathrm{Be}$ provide a qualitatively wrong energy distribution in the " $T$ " system. The situation is similar for the angular distribution in the "Y" system.

This "softness" of the ${ }^{6} \mathrm{Be}$ system makes it a very complicated object to study. The basis sizes that provide convergence for the energy and width are far from sufficient for calculations of momentum distributions. This is a feature that we probably do not face in heavier $2 p$ emitters as the Coulomb interaction in the core- $p$ channel plays a more dominant role in the decay dynamics.

The radial convergence of the energy distributions is illustrated in Fig. 13. Calculations with $\rho_{\max }<300 \mathrm{fm}$ are clearly insufficient to stabilize the distribution. However, by $\rho_{\max }=$ $800 \mathrm{fm}$, the distributions seem to be well converged. Could there be some noticeable modifications of the distributions due to further propagation in the long-range Coulomb field? This question was analyzed in Ref. [18] for ${ }^{45} \mathrm{Fe}$ using the classical trajectory approach. The complete stabilization takes place in ${ }^{45} \mathrm{Fe}$ at $\rho \sim(3-6) \times 10^{4} \mathrm{fm}$, with a major part of the effect originating at $\rho \lesssim 1 \times 10^{4} \mathrm{fm}$. The decay energies of ${ }^{6} \mathrm{Be}$ and ${ }^{45} \mathrm{Fe}$ g.s. are similar and the core- $p$ Coulomb interaction is $\sim 12$ times weaker in ${ }^{6} \mathrm{Be}$. Therefore, the majority of the long-range effects should be taken into account in calculations with $\rho_{\max } \sim 1000 \mathrm{fm}$. The ${ }^{6} \mathrm{Be}$ calculations of this work were typically done with $\rho_{\max }=1200 \mathrm{fm}$.

\section{Structure of the ${ }^{6} \mathrm{He}$ and ${ }^{6} \mathrm{Be}$ g.s.}

From another point of view, the "softness" of ${ }^{6} \mathrm{Be}$ system can be seen as a benefit. The high sensitivity of the observables to the details of the model ingredients increase our ability to discriminate these features and hence improve our ability to elucidate the details of the nuclear structure.

Detailed information about the ${ }^{6} \mathrm{He}$ and ${ }^{6} \mathrm{Be}$ g.s. WFs is provided in Table I. In general, there is a high degree of isobaric symmetry between the ${ }^{6} \mathrm{He}$ and ${ }^{6} \mathrm{Be}$ WFs in the internal region. However, this is not true for the $K=0$ component, which differs the most. The reason for this is shown in Fig. 3 where the magnitude of the $K=0 \mathrm{WF}$ component in ${ }^{6} \mathrm{Be}$ in the asymptotic region is comparable to its magnitude in the nuclear interior. Hence the nuclear boundary is not defined for this component in ${ }^{6} \mathrm{Be}$. This is also seen in Table VI, which provides the information about the WF in approximate "shell model" terms. After looking at the radial behavior of the WFs' components in Fig. 3, we find that the concept of isobaric symmetry is relevant here strictly speaking only for the most interior region of the WF $(\rho<4-5 \mathrm{fm})$. Beyond this point the radial behaviors of the ${ }^{6} \mathrm{He}$ and ${ }^{6} \mathrm{~B}$ WF's differ drastically.

The weights of the components in Tables I and VI are in very good relative agreement for the different potential sets P1-P3. Evidently these major features of the structure are not that sensitive to the fine details of the interactions.

It can be seen that the fractional partial widths $\Gamma_{i} / \Gamma$ of the ${ }^{6} \mathrm{Be}$ WF components in Table I are drastically different as compared to weights $N_{i}$ in the internal region. This is a reflection of the complicated dynamics in $2 p$ decays, the 
TABLE II. Radial properties of the ${ }^{6} \mathrm{He}$-g.s. WF and some observables obtained for the ${ }^{6} \mathrm{He}$ and the ${ }^{6} \mathrm{Be}$ g.s. with potentials $\mathrm{P} 1, \mathrm{P} 2$, and $\mathrm{P} 3$.

\begin{tabular}{lcccc}
\hline \hline Value & $\mathrm{P} 1$ & $\mathrm{P} 2$ & $\mathrm{P} 3$ & Exp. \\
\hline$\langle\rho\rangle(\mathrm{fm})$ & 5.088 & 5.156 & 5.491 & \\
$\left\langle r_{N N}\right\rangle(\mathrm{fm})$ & 4.482 & 4.502 & 4.884 & \\
$\left\langle r_{c N}\right\rangle(\mathrm{fm})$ & 4.113 & 4.172 & 4.430 & \\
$\left\langle r_{N}\right\rangle(\mathrm{fm})$ & 3.211 & 3.248 & 3.469 & \\
$\left\langle r_{c}\right\rangle(\mathrm{fm})$ & 1.321 & 1.171 & 1.232 & \\
$r_{\text {mat }}(\mathrm{fm})$ & 2.396 & 2.421 & 2.540 & $2.30 \pm 0.07[33]$ \\
& & & & $2.48 \pm 0.03[34]$ \\
$r_{\text {ch }}{ }^{\mathrm{a}}(\mathrm{fm})$ & 2.103 & 2.012 & 2.048 & $2.054 \pm 0.014[35]$ \\
$r_{\text {ch }}{ }^{\mathrm{b}}(\mathrm{fm})$ & 2.113 & 2.043 & 2.079 & $2.068 \pm 0.011[36]$ \\
$B_{G T}\left({ }^{6} \mathrm{He} \rightarrow{ }^{6} \mathrm{Li}\right)$ & 5.004 & 5.058 & 4.930 & $4.745 \pm 0.009[15]$ \\
$\Delta E_{\text {coul }}(\mathrm{MeV})$ & 2.351 & 2.302 & 2.111 & $2.344[15]$ \\
$\Gamma\left({ }^{6} \mathrm{Be}\right.$ g.s. $)(\mathrm{keV})$ & 98 & 112 & 154 & $92 \pm 6[15]$ \\
\hline \hline
\end{tabular}

${ }^{\text {a Theoretical values in this row are obtained using the generally }}$ accepted value for the neutron charge radius $r_{\mathrm{ch}}^{2}(n)=-0.1161 \mathrm{fm}^{2}$. ${ }^{\mathrm{b}}$ Theoretical values in this row are obtained using $r_{\mathrm{ch}}^{2}(n)=0.012 \mathrm{fm}^{2}$ [36].

WFs are strongly "rearranged" in the sub-barrier region by the long-range Coulomb pairwise fields. The ${ }^{6} \mathrm{He}$ and ${ }^{6} \mathrm{Be}$ spatial correlation densities are shown in Fig. 9. The behavior of the correlation densities is nearly identical in the internal region, while in the asymptotic region for ${ }^{6} \mathrm{Be}$, we can clearly see how this "rearrangement" is taking place. Comparing the different potential sets P1-P3 in Table I, we see that P1 and P2 calculations are almost identical, while the major partial widths in P3 case differ strongly. We conclude that the decay dynamics is mainly defined by the core- $p$ interaction.

Geometric properties of the ${ }^{6} \mathrm{He}$ g.s. WF and several observables obtained for the ${ }^{6} \mathrm{He}$ and ${ }^{6} \mathrm{Be}$ g.s. are shown in Table II. The root-mean-square values are given for $\rho, r_{N N}$ (distance between the valence nucleons), $r_{c N}$ (distance between the nucleon and core), $r_{N}$ (distance between the valence nucleon and center-of-mass), $r_{c}$ (distance between the core and center-of-mass). The differences between these geometric characteristics for $\mathrm{P} 1$ and $\mathrm{P} 2$ are typically around $1 \%$. In the case of $\mathrm{P} 3$, the differences are significantly larger. The $B_{\mathrm{GT}}$ values for ${ }^{6} \mathrm{He}$ (g.s.) $\rightarrow{ }^{6} \mathrm{Li}$ (g.s.) $\beta$-decay obtained with P1-P3 also agree within $1.5 \%$, but all differ more from the experimental value. Here, the "experimental" $B_{\mathrm{GT}}$ values are obtained using the ${ }^{6} \mathrm{He}$ lifetime $\tau_{1 / 2}=806.7 \pm 1.5 \mathrm{~ms}$ [15] and with the $\beta$-decay constants of $f t\left(0^{+} \rightarrow 0^{+}\right)=3072.40 \mathrm{~s}$ and $\lambda=1.268$. It has already been discussed in the literature that the 4-7\% disagreement here could be connected with both the WF quality and the renormalization of the weak constant [28]. Therefore, we can give no definite conclusion about the quality of the models here.

The next most precisely known characteristic for ${ }^{6} \mathrm{He}$ is its charge radius. Recent studies have defined $r_{\text {ch }}$ with increasing precision $[35,36]$. The relative uncertainty of this value is now about $0.5 \%$, while variations in the calculated values are around $4 \%$ for P1-P3. However, comparisons of our theoretical values to experiment are not completely model independent. The calculated charge radius of ${ }^{6} \mathrm{He}$ is noticeably sensitive to the neutron charge radius:

$$
r_{\mathrm{ch}}^{2}\left({ }^{6} \mathrm{He}\right)=r_{\mathrm{ch}}^{2}(\alpha)+r_{\mathrm{ch}}^{2}(n)+\left\langle r_{c}\right\rangle^{2} .
$$

As the $r_{\mathrm{ch}}^{2}(n)$ value is inferred theoretically, rather than measured experimentally, this means that there exists considerable theoretical uncertainty in the determination of the charge radii that is not connected with the quality of the ${ }^{6} \mathrm{He} W F$ itself. According to our estimates, this uncertainty can be as large as $2 \%$. This fact somewhat relieves the constraints on the WF connected with this observable. One can see in Table II that the $\mathrm{P} 2$ and $\mathrm{P} 3$ calculations, containing realistic $N N$ potentials can be regarded as consistent with the experiment.

The matter radius of ${ }^{6} \mathrm{He}$ is defined in the cluster model using the matter radius of the $\alpha$ particle:

$$
6 r_{\text {mat }}^{2}\left({ }^{6} \mathrm{He}\right)=4 r_{\text {mat }}^{2}(\alpha)+\langle\rho\rangle^{2} .
$$

The value $r_{\text {mat }}(\alpha)=1.464 \mathrm{fm}$ is derived from the charge radius $r_{\mathrm{ch}}(\alpha)=1.671$ using the neutron and proton charge radii; $r_{\mathrm{ch}}^{2}(n)=-0.1161 \mathrm{fm}^{2}, r_{\mathrm{ch}}(p)=0.875 \mathrm{fm}$. The experimental data on matter radii have a large systematic uncertainty. This is probably the reason for the controversial signal obtained in different experiments (see two examples in Table II). This observable thus far does not seem to be able to discriminate between theoretical models.

The Coulomb shift $\Delta E_{\text {coul }}$ and the ${ }^{6}$ Be g.s. width obtained with $\mathrm{P} 1$ and $\mathrm{P} 2$ are in a good agreement with experiment. Some overestimation of the width in the three-body cluster model can be expected due to the admixture of different (not belonging to the three-cluster model space) configurations in ${ }^{6} \mathrm{Be} W F$. The weight of such admixtures can be estimated as 6-14\%, based on the P1 and P2 widths. However, the Coulomb shift and width obtained with P3 are clearly not acceptable. Our overall feeling is that the cumulative information on the ${ }^{6} \mathrm{He}$ and ${ }^{6} \mathrm{Be}$ g.s. is sufficient to choose P2 as the only acceptable potential set.

\section{THEORETICAL DISCUSSION}

As we have already mentioned, most of the attention in the studies of the $A=6$ isobars has been paid to ${ }^{6} \mathrm{He}$. Even in the studies of ${ }^{6} \mathrm{Be}$, there are only a few works that studied its width. In addition, there has been only limited studies of the ${ }^{6} \mathrm{Be}$ g.s. decay correlations. The first consistent calculations of the ${ }^{6} \mathrm{Be}$ three-body decay width were performed in Ref. [37] using the integral formalism. In Refs. [16,17], the quantum-mechanical formalism for two-proton radioactivity and Coulombic three-body decay studies was developed. In these articles, the integral formalism was criticized in application to the decays of systems with strong three-body Coulomb interactions and a more preferable way to calculate widths was proposed [see, Eq. (11)]. The value $\Gamma=90 \mathrm{keV}$ was obtained in Ref. [16] with the P1 potential $\left(K_{\max }=20\right)$, which as we can see in Fig. 6, is reasonably well converged.

In our approach, the effects of antisymmetrization are taken into account in a simplified way. However, there are studies that treated the ${ }^{6} \mathrm{Be}$ decay as a six-body problem. In resonating-group-method calculations [38], the ${ }^{6} \mathrm{Be}$ width of $\Gamma=160 \mathrm{keV}$ for $E_{3 r}=1.52 \mathrm{MeV}$ was found using the complex scaling method. Scaling this value to the experimental $2 p$ decay energy, with the help of Fig. 5, we obtain 
$\Gamma=125 \mathrm{keV}$, which is considerably larger than the experimental value. An interesting algebraic method was developed for studies of ${ }^{6} \mathrm{Be}$ decay in Ref. [39]. Here, the hyperspherical decomposition is used for the WF both in the internal region (six-body HHs) and in the asymptotic region (three-body cluster $\mathrm{HHs}$ ). A calculated width of $\Gamma=72 \mathrm{keV}$ was obtained for $E_{3 r}=1.172 \mathrm{MeV}$ that scales to $\Gamma=110 \mathrm{keV}$ at the experimental $2 p$ decay energy. In addition, we can expect a $10-15 \%$ reduction due to the absence of the $S=1$ component in these calculations. This component is important in the internal region but does not contribute to the width significantly. In addition, we can also expect roughly a factor of 2 increase due to the small basis size $\left(K_{\max }=10\right)$ used in the asymptotic region in Ref. [39]. According to Fig. 6, with $K_{\max }=10$ we can expect to find at most $60 \%$ of the width. It seems that Ref. [39] is more a concept demonstration rather than a realistic calculation. Therefore at present, it is not possible to draw any conclusions about importance of six-body effects in calculations of the ${ }^{6} \mathrm{Be}$ decay properties.

The width of the ${ }^{6} \mathrm{Be}$ g.s. was calculated in Ref. [40] via a method analogous to ours (hyperspherical harmonics), but having certain technical differences. An approximate treatment of the $3 \rightarrow 3$ scattering is introduced in this work and the width is extracted from the energy behavior of the phase shifts. The width obtained was $\Gamma=65 \mathrm{keV}$ for $E_{3 r}=1.26 \mathrm{MeV}$ that scales to $\Gamma=84 \mathrm{keV}$ at the experimental $2 p$ decay energy. It can be found in Ref. [40] that the calculation does not seem to have converged. If we extrapolate from $\Gamma=84 \mathrm{keV}$ using the convergence curves for P2 and P3 (see Fig. 5), then the value $\Gamma=110 \mathrm{keV}$ is obtained, which is in a good agreement with our $\mathrm{P} 2$ result.

An important result of the present work is a clear demonstration that any approach purporting to give satisfactory description of the ${ }^{6} \mathrm{Be}$ g.s. decay properties should have a large enough "dynamic range" both in radial and functional spaces (see Table III).

Our calculations demonstrate a noticeable sensitivity of the observables in the decay of the ${ }^{6} \mathrm{Be}$ g.s. to the ingredients of the model. Table II demonstrates that this sensitivity is enhanced in ${ }^{6} \mathrm{Be}$ compared to ${ }^{6} \mathrm{He}$. Typical variations of the observables for ${ }^{6} \mathrm{He}$ are $0.5-4 \%$, while in ${ }^{6} \mathrm{Be}$ there is about a $60 \%$ difference between the widths calculated with P1 and P3. The tunneling process can be seen as a kind of a "quantum amplifier," which strongly emphasizes minor features in the structure. For that reason, it is possible that the indirect experimental probe of the ${ }^{6} \mathrm{Be}$ decay is a more sensitive tool to get access to the halo properties of ${ }^{6} \mathrm{He}$ than direct investigations of the ${ }^{6} \mathrm{He}$ itself.

TABLE III. Minimal dynamical ranges of calculations required to provide reasonably converged different observables for ${ }^{6} \mathrm{Be}$. Different basis sizes are required for simplistic BJ and realistic GPT potentials in the $p-p$ channel.

\begin{tabular}{lccc}
\hline \hline Value & $E_{3 r}$ & $\Gamma$ & Distributions \\
\hline$\rho_{\max }(\mathrm{fm})$ & 20 & 60 & 300 \\
$K_{\max }(\mathrm{SBB}+\mathrm{BJ})$ & 16 & 30 & 80 \\
$K_{\max }(\mathrm{SBB}+\mathrm{GPT})$ & 40 & 70 & 110 \\
\hline \hline
\end{tabular}

We are referring to precision measurements of the correlations in ${ }^{6} \mathrm{Be}$ decay that are discriminative with respect to the fine details of the momentum distributions. The quality of the new experimental results, reported on in Sec. VI, approach that required to make this discrimination.

\section{EXISTING EXPERIMENTAL KNOWLEDGE ABOUT ${ }^{6}$ Be}

Very precise results about the energy and width of the ${ }^{6} \mathrm{Be}$ g.s. were obtained already in the early studies: $E_{T}=$ 1383(40) $\mathrm{keV}, \Gamma=140(40) \mathrm{keV}$ [41], $E_{T}=1371(6) \mathrm{keV}$, $\Gamma=89(6) \quad \mathrm{keV} \quad$ [42], and $E_{T}=1372(9) \quad \mathrm{keV}, \quad \Gamma=$ 95(28) $\mathrm{keV}$ [43]. The currently accepted value of the width $\Gamma=92(6) \mathrm{keV}[15]$ is based on the above results and it has not changed since the early 1970 s.

The first measurements of ${ }^{6} \mathrm{Be}$ decay correlations were made in Ref. [44], see Fig. 14(a). They determined the energy spectrum of $\alpha$ particles reconstructed in the ${ }^{6} \mathrm{Be}$ center-of-mass frame. For the ${ }^{6} \mathrm{Be}$ g.s. events, this spectrum can also be presented as the correlation spectrum between two protons. The authors could not fit the data using simplistic decay scenarios (phase volume, diproton decay, simultaneous emission of $p$-wave protons) and concluded: “. . .no incoherent sum of the processes considered here will fit the data. Perhaps a full three-body computation is necessary to understand the energy spectrum." We think that this point of Ref. [44] is very important and it finds confirmation in our work on the new level of sophistication (see, e.g., discussion in Sec. VIII).

This ground-state decay, as well as decays of the $2^{+}, T=0$ states of the $A=6$ isobars, were further investigated in the series of works by the Kurchatov Institute group ([13,30,31] and Refs. therein), see Fig. 14(b). They developed a method

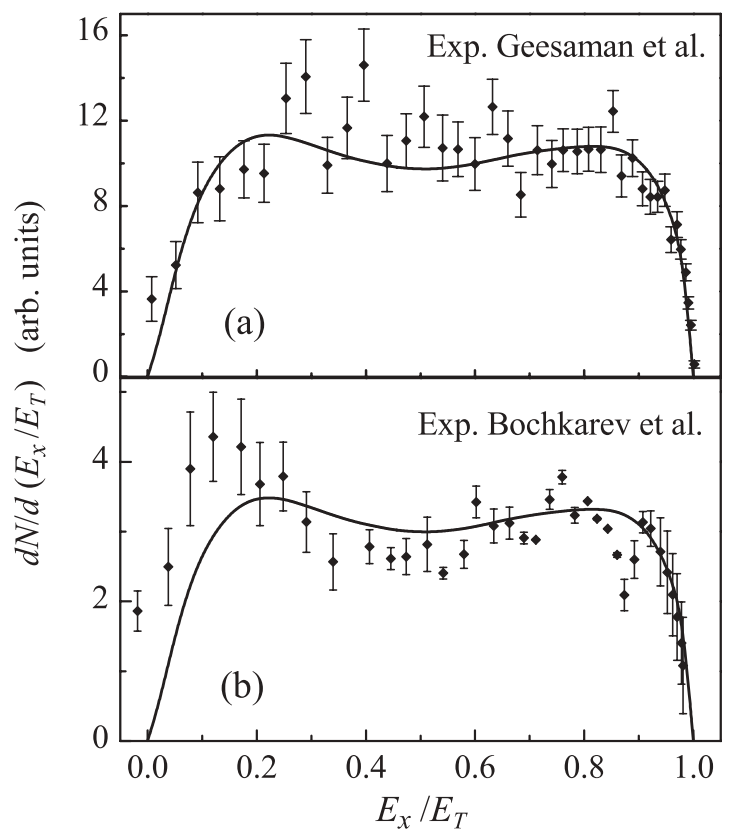

FIG. 14. Experimental energy distributions between the protons in the decay of ${ }^{6} \mathrm{Be}$ measured in (a) Ref. [44] and (b) Ref. [31]. The theoretical prediction (P1) is provided only to guide the eye, as the required experimental corrections are not known. 
of analyzing the $p$ - $p$ correlations in the framework of a three-body partial-wave decomposition and applied this to the three-body decays of light nuclei [31,32]. In particular, the first kinematically complete study of ${ }^{6} \mathrm{Be}$ proved the existence of three-particle $p+p+\alpha$ correlations with total spin of the proton-proton subsystem of $S(p-p)=1$ and $S(p-p)=0[13,31]$ that matched the three-body components found theoretically in the $p$-shell structure of ${ }^{6} \mathrm{Be}$ [28]. One of the important results for the ${ }^{6} \mathrm{Be}$ g.s. was the realization that $S(p-p)=0$ and $S(p-p)=1$ components of the WF should produce very different correlation patterns. The presence of an "admixture" of $S(p-p)=1$ component to the WF was demonstrated by an experiment performed with special kinematics. In these works, the concept of "democratic decay" was coined. This describes the specific decay mode for threebody systems, when the events are not highly focused in narrow kinematic regions but are distributed broadly ("democracy" among different kinematic regions). "Democratic decay" is now a popular term for this class of phenomena, but the correlations in ${ }^{6} \mathrm{Be}$ decay have never been studied since that time. The spectra shown in Figs. 14(a) and 14(b) are not in complete agreement with each other. Furthermore, there are large statistical uncertainties and the geometry of experiments may cause cuts in kinematic space that make comparison with the theory difficult. It is clear that better experimental data on ${ }^{6}$ Be decay are needed.

\section{NEW EXPERIMENT}

\section{A. Experimental method}

The Texas A\&M University K500 cyclotron facility was used to produce a 200-pnA beam of ${ }^{10} \mathrm{~B}$ at $E / A=15.0 \mathrm{MeV}$. This primary beam impinged on a hydrogen gas cell held at a pressure of $2 \mathrm{~atm}$ and kept at liquid-nitrogen temperature. A secondary beam of $E / A=10.7 \mathrm{MeV}{ }^{10} \mathrm{C}$ was produced through the ${ }^{10} \mathrm{~B}(p, n){ }^{10} \mathrm{C}$ reaction and separated from other reaction products using the MARS spectrometer [45]. This secondary beam, with intensity of $2 \times 10^{5} \mathrm{~s}^{-1}$, purity of $99.5 \%$, an energy spread of $3 \%$, and a spot size of $3.5 \times 3.5 \mathrm{~mm}$ was inelastically excited due to interactions with $14.1 \mathrm{mg} / \mathrm{cm}^{2}$ $\mathrm{Be}$ and $13.4 \mathrm{mg} / \mathrm{cm}^{2} \mathrm{C}$ targets. Ground-state ${ }^{6} \mathrm{Be}$ fragments were created from the $\alpha$ decay of these excited ${ }^{10} \mathrm{C}$ particles. Following the decay of the ${ }^{6} \mathrm{Be}$ g.s. fragment, the final exit channel is $2 p+2 \alpha$.

The four decay products were detected in an array of four Si $E-\Delta E$ telescopes located in a plane $14 \mathrm{~cm}$ downstream of the target. The telescopes, part of the HiRA array [46], consisted of a $65-\mu \mathrm{m}$-thick, single-sided Si-strip $\Delta E$ detector followed by a 1.5 -mm-thick, double-sided Si strip $E$ detector. All Si detectors were $6.4 \times 6.4 \mathrm{~cm}$ in area with their positionsensitive faces divided into 32 strips. The telescopes were positioned in a square arrangement with each telescope offset from its neighbor to produce a small, central, square hole through which the unscattered beam passed. With this arrangement, the angular range from $\theta=1.3$ to $7.7^{\circ}$ was covered. More details of the experimental arrangement can be found in Ref. [9].

\section{B. Monte Carlo simulations}

Monte Carlo simulations of the experiment were performed to determine the experimental bias and to understand the effects of the gates applied to remove unwanted $2 p+2 \alpha$ events. The simulations included the $\alpha$ decay of the parent ${ }^{10} \mathrm{C}$ fragments and the ${ }^{6} \mathrm{Be}$ ground-state energy and width were taken from Ref. [15]. The correlations between the ${ }^{6} \mathrm{Be}$ decay products were sampled according to the theory of Sec. III B. The effects of energy loss and small-angle scattering of all the decay products were considered following Refs. [47,48].

Simulated events were passed through a detector filter and the effects of the position and energy resolution of the detector were added. The "detected" simulated events were subsequently analyzed in the same manner as the experimental data. The velocity, excitation-energy, and angular distributions of the parent ${ }^{10} \mathrm{C}$ states were chosen such that the secondary distributions that passed the detector filter were consistent with the experimental results. Similar simulations for other decay modes were found to reproduce the experimental resolution [9].

\section{Event selection}

Apart from $\alpha-{ }^{6}$ Be g.s. decay, there are many other ${ }^{10} \mathrm{C}$ decay modes that lead to the $2 p+2 \alpha$ exit channel and thus the detected events must be suitably gated to remove these unwanted decays. Of particular importance is the rejection of the large yield of decays where the ${ }^{10} \mathrm{C}$ fragment undergoes two-proton decay (either sequential through ${ }^{9} \mathrm{~B}$ or prompt) leading to the creation of an ${ }^{8} \mathrm{Be}$ g.s. [10]. These events can readily be identified from the correlations between the two $\alpha$ particles. The distribution of relative energy $\left(E_{\text {rel }}^{\alpha \alpha}\right)$ between the two $\alpha$ particles contains a strong, narrow peak corresponding to ${ }^{8}$ Be-g.s. decay [9]. This peak has a full width at half maximum (FWHM) of $38 \mathrm{keV}$ and sits on a negligible background [9], thus allowing for a clean rejection of these events with the gate $E_{\mathrm{rel}}^{\alpha \alpha}<0.2 \mathrm{MeV}$. Our Monte Carlo simulations suggests this gate has essentially no significant effect on true $\alpha-{ }^{6} \mathrm{Be}$ g.s. decays with only $0.01 \%$ of detected events being rejected.

The remaining events have contributions from $\alpha-{ }^{6} \mathrm{Be}$ g.s. and $p-{ }^{9} \mathrm{~B}\left(E^{*}=2.43 \mathrm{MeV}\right)$ decays [9]. The latter ${ }^{9} \mathrm{~B}$ excited state does not decay through ${ }^{8} \mathrm{Be}$ g.s. but undergoes a three-body decay like the ${ }^{6} \mathrm{Be}$ ground state. For both of these decays modes, there is a difficultly in trying to find the intermediate state (either ${ }^{6} \mathrm{Be}$ or ${ }^{9} \mathrm{~B}$ ) as there are two possible ways to construct this fragment from the detected $2 p+2 \alpha$ exit channel. Let us concentrate on the ${ }^{6} \mathrm{Be}$ g.s. fragments first where we must determine which of the two detected $\alpha$ particles was the one initially emitted from the ${ }^{10} \mathrm{C}$ parent and which was produced in the decay of ${ }^{6} \mathrm{Be}$. To this end, the ${ }^{6} \mathrm{Be}$ excitation energy for the two ways of constructing the ${ }^{6} \mathrm{Be}$ fragment are determined and ordered according to their maximum and minimum values; $E^{*}\left({ }^{6} \mathrm{Be}\right)_{\max }$ and $E^{*}\left({ }^{6} \mathrm{Be}\right)_{\min }$. A two dimensional plot of these two excitation energies is shown in Fig. 15. A prominent ridge centered around $E^{*}\left({ }^{6} \mathrm{Be}\right)_{\min }=0$ corresponding to ${ }^{6} \mathrm{Be}$ g.s. decay is clearly visible. For those events in this ridge structure, the identification of which $\alpha$ particle was produced in ${ }^{6} \mathrm{Be}$ decay is clearly the one associated 


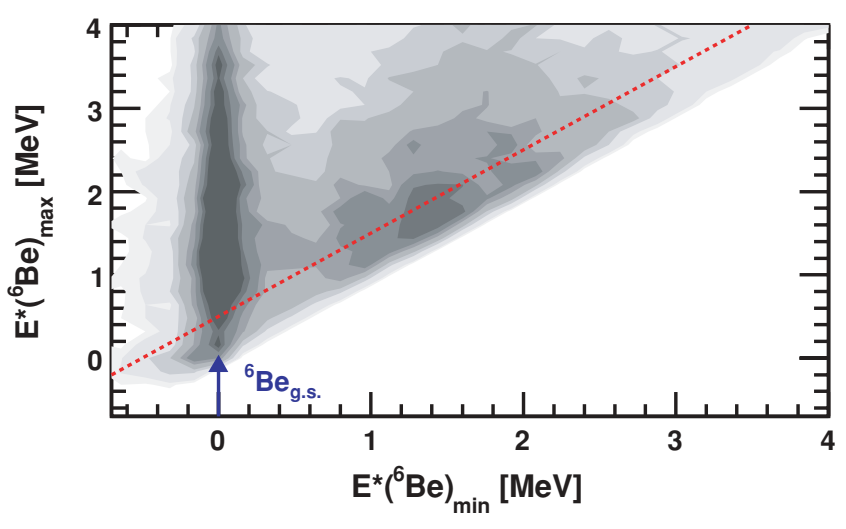

FIG. 15. (Color online) Contour plot showing the distribution of the two possible ${ }^{6} \mathrm{Be}$ excitation energies that can be associated with the two $2 p+\alpha$ subevents ordered by their maximum and minimum values. The dashed line indicates the threshold for which correct identification of the $2 p+\alpha$ subevent associated with ${ }^{6} \mathrm{Be}$ decay is achieved in the simulations. The ridge associated with the ${ }^{6} \mathrm{Be}$ g.s. decay is indicated by the arrow.

with $E^{*}\left({ }^{6} \mathrm{Be}\right)_{\min }$ when $E^{*}\left({ }^{6} \mathrm{Be}\right)_{\max } \gg E^{*}\left({ }^{6} \mathrm{Be}\right)_{\min }$. However when $E^{*}\left({ }^{6} \mathrm{Be}\right)_{\max } \sim E^{*}\left({ }^{6} \mathrm{Be}\right)_{\min }$ the Monte Carlo simulations indicate that misidentifications will occur. These simulations suggests that for $E^{*}\left({ }^{6} \mathrm{Be}\right)_{\max }-E^{*}\left({ }^{6} \mathrm{Be}\right)_{\min }=0.5 \mathrm{MeV}$, the probability of misidentifying the $\alpha$ particles is $0.03 \%$. This condition is indicated in Fig. 15 by the dashed line and only events above this line were used in the subsequent analysis of the experimental data. One can see from Fig. 15 that this condition does not significantly cut into the ridge structure and the Monte Carlo simulations suggests we lose $4.7 \%$ of the remaining $\alpha-{ }^{6} \mathrm{Be}$ g.s. events with this gate.

The remaining ridge structure still sits on a background. Part of this background can be traced to ${ }^{10} \mathrm{C} \rightarrow p+{ }^{9} \mathrm{~B}\left(E^{*}=\right.$ $2.43 \mathrm{MeV})$ decays. These events can be identified from $E^{*}\left({ }^{9} \mathrm{~B}\right)_{\max }$ and $E^{*}\left({ }^{9} \mathrm{~B}\right)_{\min }$ information in a manner similar to the $\alpha-{ }^{6} \mathrm{Be}$ g.s. events. A ridge structure also is evident in this case and it also sits on an non-negligible background, which in turn has contributions from $\alpha-{ }^{6} \mathrm{Be}$ g.s. decay. Although one cannot completely separate all $p-{ }^{9} \mathrm{~B}$ and $\alpha-{ }^{6} \mathrm{Be}$ events, we do reject events in the $E^{*}\left({ }^{9} \mathrm{~B}\right)_{\text {min }}$ ridge structure. This results in a slightly diminished yield of true $\alpha-{ }^{6} \mathrm{Be}$ g.s. events, but more importantly, it reduces the relative background under the ${ }^{6} \mathrm{Be}$ ridge structure shown in Fig. 15. The Monte Carlo simulations suggests only $2.7 \%$ of the remaining true $\alpha-{ }^{6}$ Be g.s. events were rejected by this condition.

The distribution of $E_{T}$ for the final selection of events is shown in Fig. 16 by the data points. The FWHM width of the peak associated with the ${ }^{6} \mathrm{Be}$ g.s. is $220 \mathrm{keV}$, which is larger than the intrinsic value of $\Gamma=92 \mathrm{keV}$ due to the detector resolution. The solid curve indicates the simulated distribution after a smooth background contribution (dashed curve) is added. This simulated distribution reproduces the experimental results quite well, confirming that the Monte Carlo simulations correctly model the experimental resolution. Figure 16 also shows the gate $G 6$ used to select ${ }^{6} \mathrm{Be}$ g.s. fragments and the two gates, $G_{B 1}$ and $G_{B 2}$ that, when combined,

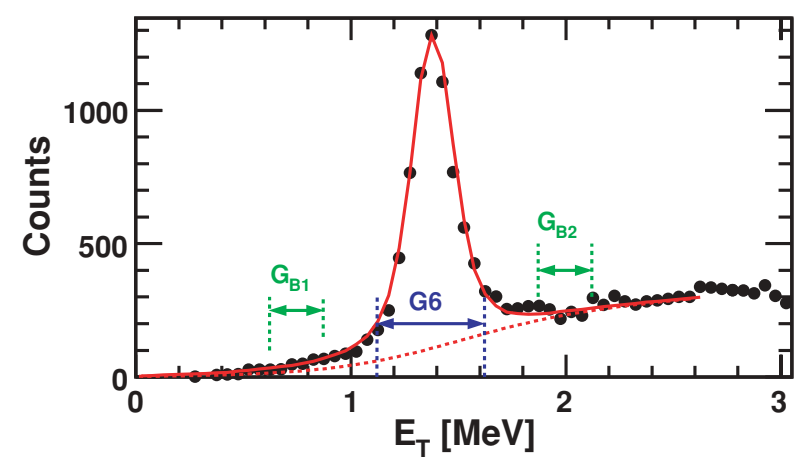

FIG. 16. (Color online) The experimental distribution of $E_{T}$ for selected events is shown by the data points. The solid curve indicates the distribution predicted by the Monte Carlo simulation with the addition of a smooth background (dashed curve). The $G 6$ gate used to select ${ }^{6} \mathrm{Be}$ g.s. events and the two gates $\left(G_{B 1}, G_{B 2}\right)$, used to estimate the background under the peak, are indicated.

were used to estimate the background in the $G 6$ gate. In all subsequent results, this background has been subtracted.

The excitation-energy distribution of ${ }^{10} \mathrm{C}$ fragments associated with the selected events is shown in Fig. 17. There is localized strength around $E^{*}\left({ }^{10} \mathrm{C}\right)=7 \mathrm{MeV}$ and a continuous distribution up to approximately $15 \mathrm{MeV}$. Thus many ${ }^{10} \mathrm{C}$ excited states are contributing to the detected ${ }^{6} \mathrm{Be}$ g.s. yield.

\section{COMPARISON OF THEORY AND EXPERIMENT}

Comparisons of experimental and predicted correlations in both the "T" and "Y" Jacobi systems are shown in Fig. 18. The experimental results [Figs. 18(a) and 18(c)] have been background subtracted and, for the predicted distributions [Figs. 18(b) and 18(d)], the effects of the detector resolution and bias have been incorporated via the Monte Carlo simulations. In subsequent plots, the simulated results have been normalized to the same number of counts as for the experimental data. In determining the Jacobi coordinates, there are two ways of choosing the order of the proton. For the experimental events, Jacobi coordinates were determined for both of these ways and thus each event contributes two counts to the spectra. For "T" system, this forces the $\cos \left(\theta_{k}\right)$ distribution to be symmetrized around $\cos \left(\theta_{k}\right)=0$. General

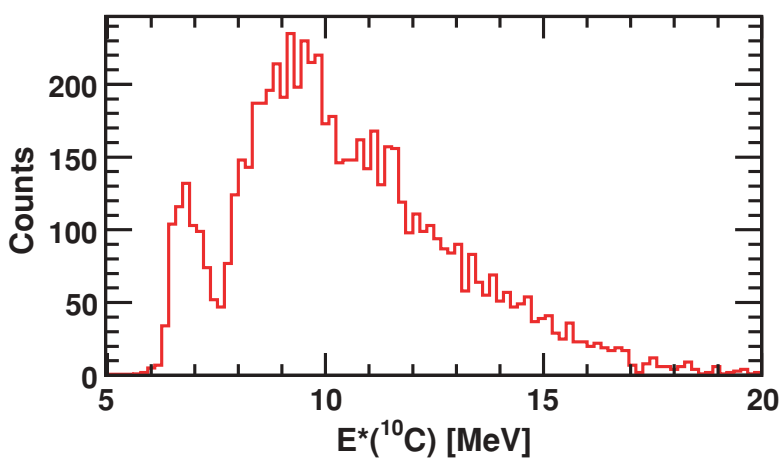

FIG. 17. (Color online) Experimental distribution of ${ }^{10} \mathrm{C}$ excitation energy for $\alpha-{ }^{6} \mathrm{Be}$ g.s. events selected in this study. 
(a) "T",exp

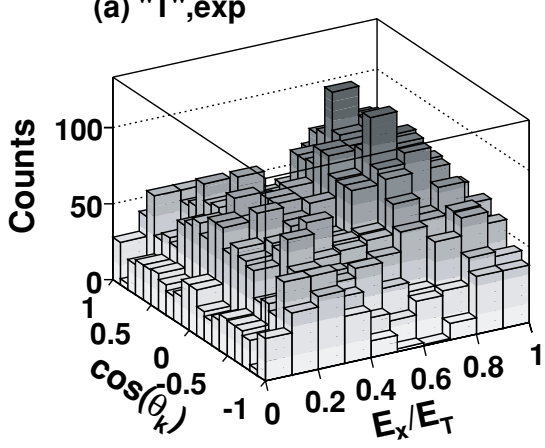

(c) "Y", exp

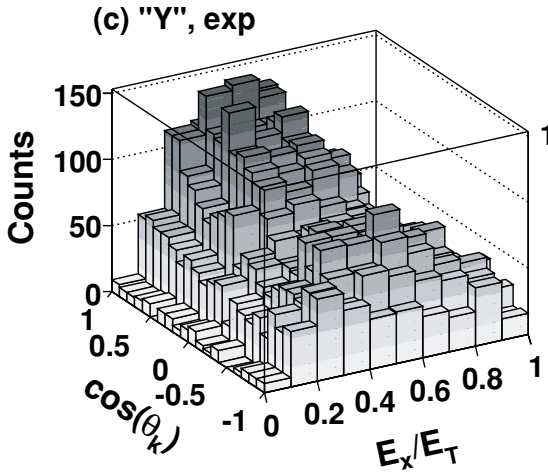

(b) "T", theory

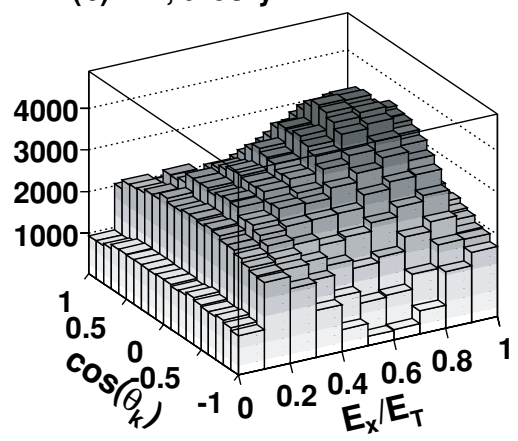

(d) "Y", theory

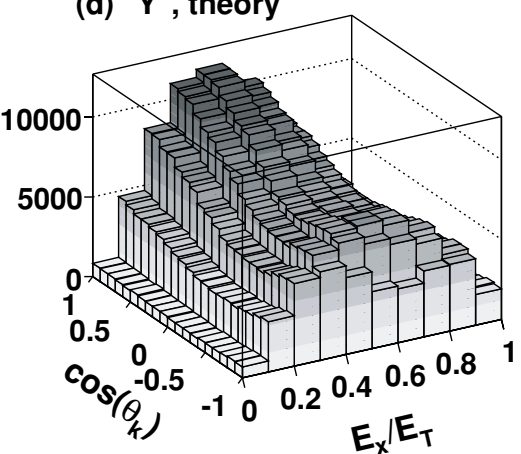

FIG. 18. Comparison of (a and c) experimental (exp) and ( $b$ and d) theoretical correlations for the ${ }^{6} \mathrm{Be}$ g.s. decay presented in both the (a and b) " $\mathrm{T}$ " and (c and d) "Y" Jacobi systems. The theoretical distributions include the effects of the detector bias and resolution via the Monte Carlo simulations. overall agreement between theory and experiment is found, although statistical fluctuations are the limiting factor for the experimental data.

To allow for a more detailed comparison, we compare projections of the correlations on both the $E_{x} / E_{T}$ and $\cos \left(\theta_{k}\right)$ axes in Fig 19. The experimental data are indicated by the data points while the dashed and solid curves show the predictions before and after the simulated bias of the
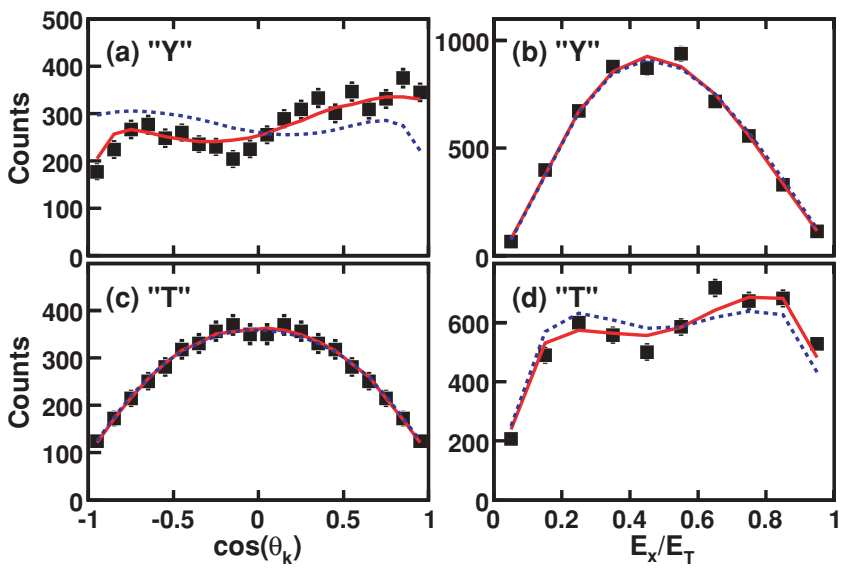

FIG. 19. (Color online) Comparison of the experimental (data points) and predicted (curves) distributions of $\cos \left(\theta_{k}\right)$ and $E_{x} / E_{T}$ in the (c and d) "T" and (a and b) "Y" Jacobi systems. The blue dashed curves show the primary predicted distributions while the red solid curves include the effect of the detector bias and resolution. The theoretical results were obtained with the $\mathrm{P} 2$ potential. experimental apparatus is included. Interestingly, the "soft" observables (energy distribution in the "T" system and the angular distribution in the "Y" system) that have the most sensitivity to the ingredients of the theoretical calculations and its numerical implementation also have the largest bias induced by the detector apparatus. The other projected distributions (angular distribution in " $\mathrm{T}$ " and energy distribution in "Y") are practically unaffected by the detector response.

The same comparison of theory and data for all three potentials P1-P3 is shown in Fig. 20. All three sets of predictions reproduce the experimental data reasonably well. To highlight more details of the correlations, we show the $\cos \left(\theta_{k}\right)$ distributions gated on three equal regions of $E_{x} / E_{T}$ in Fig. 21 for the "T" and "Y" Jacobi systems. Reasonable agreement between the experiment (data points) and the three calculations (curves) is also found, although the P1 and P2 calculation are somewhat better. To quantify this, we determine the $\chi^{2}$ per degree of freedom $\left(\chi^{2} / v\right)$ of the theoretical fit to the two-dimensional data of Fig. 18. These values are listed in Table IV for both the "T" and "Y" systems. For a good fit we

TABLE IV. $\chi^{2}$ per degree of freedom for fits to the complete correlations data in the " $\mathrm{T}$ " and "Y" system with the three assumed potentials

\begin{tabular}{lccc}
\hline System & $\mathrm{P} 1$ & $\mathrm{P} 2$ & $\mathrm{P} 3$ \\
\hline "T" & 1.29 & 1.17 & 1.58 \\
"Y" & 1.25 & 1.14 & 1.45 \\
\hline \hline
\end{tabular}



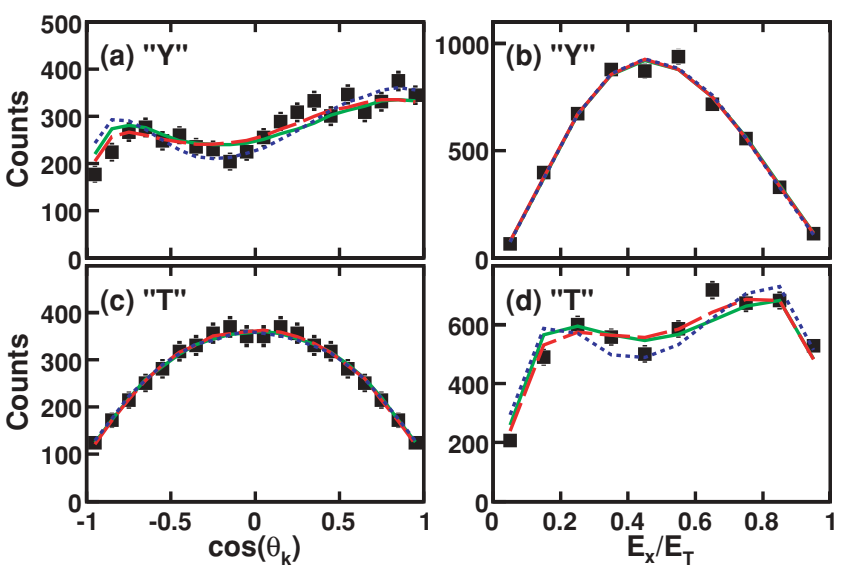

FIG. 20. (Color online) Comparison of the experimental (data points) and predicted (curves) distributions of $\cos \left(\theta_{k}\right)$ and $E_{x} / E_{T}$ in the (c and d) "T" and (a and b) "Y" Jacobi systems. The solid (green), dashed (red), and dotted (blue) curves correspond to P1, P2, and $\mathrm{P} 3$ potential sets, respectively. The effect of the detector bias and resolution is included for the theoretical curves.

need $\chi^{2} / v \sim 1$ and clearly both P1 and P2 satisfy this criteria. Again we find the P3 calculation is somewhat worse.
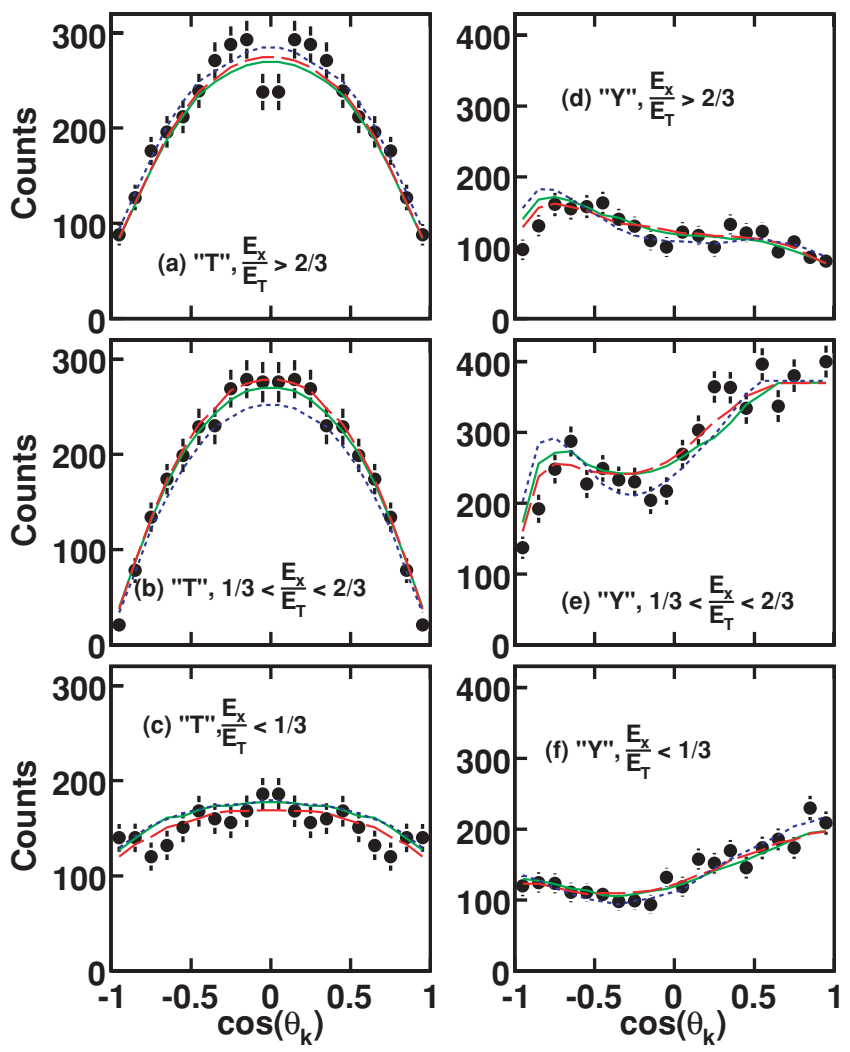

FIG. 21. (Color online) Comparison of experimental (data points) and predicted (curves) $\cos \left(\theta_{k}\right)$ distributions in the " $\mathrm{T}$ " (left) and " $\mathrm{Y}$ " (right) Jacobi systems for the indicated gates on $E_{x} / E_{T}$ parameter. The solid (green), dashed (red), and dotted (glue) curves correspond to $\mathrm{P} 1, \mathrm{P} 2$, and $\mathrm{P} 3$ potential sets, respectively. The effect of the detector bias and resolution is included.

\section{DISCUSSION OF EXPERIMENTAL DATA}

\section{A. General notes}

An important task of this work is to demonstrate a conceptual possibility of using $2 p$ correlations as a sensitive structure research tool. What do we mean here? First, data with high statistical accuracy (hundreds of events per bin in kinematic space), high acceptance, and sufficient resolution (to have minimal distortions for all parts of kinematic space) should be accessible. There have been very limited number of studies of this class for three-body decays. Second, to bridge from the internal part of our WF to the asymptotic region, we need an appropriate theoretical model. This model should be sufficiently precise to provide the quantitative description of correlations in the complete kinematic range. It was not clear in advance whether the available theoretical model provided the required precision. In this work, we have found that all reasonable potential sets P1-P3 give a good fit to the data with $\chi^{2} / v \sim 1$. Strictly speaking, this means that with the present statistical errors, we cannot make reliable discrimination among the P1-P3 cases. Nevertheless, we find it very encouraging that the $\chi^{2} / v$ is minimal for potential set $\mathrm{P} 2$, which provides the optimal agreement with the remaining data for ${ }^{6} \mathrm{He}$ and ${ }^{6} \mathrm{Be}$ (see Sec. III D). We have clearly demonstrated, at least by this one example, that there is a sensitivity of decay correlations to the minor details of interactions and consequently to details of the structure. The present experimental precision is already sufficient to become sensitive to such minor details. It is clear that with increased experimental precision, the real discriminative power of such measurements will be attained.

Important qualitative insights can also be obtained using the present experimental data and two simplistic quasiclassical models of $2 p$ emission. This is done in the next two subsections.

\section{B. On the applicability of diproton model}

The first notions about $2 p$ decay were the simplistic "diproton" picture [1,49] that is still popular [50-54]. Here the two protons are traveling under the barrier in the $S=0$ state and can be considered as a single "diproton" particle. In this model, the differential width in the "T" system is

$$
\frac{d \Gamma}{d \varepsilon}=2 \gamma_{p p}^{2} \rho\left(\varepsilon E_{T}\right) P_{0}\left(E_{T}(1-\varepsilon), r_{\mathrm{ch}}^{(d p)}, 2 Z_{\text {core }}\right) .
$$

This is a quasiclassical (QC) approach as it assumes that the "rigid" diproton propagates under the barrier along a classical trajectory. The function $P_{l}$ is a standard penetrability factor depending on the energy, channel radius, and charges and $\rho\left(\varepsilon E_{T}\right)$ is the "density of diproton states." This density has been approximated as either a "fixed-energy diproton," i.e., $\rho(E)=\delta\left(E-E_{0}\right)$ with $E_{0} \approx 50 \mathrm{keV}$ or alternatively for a "Coulomb-corrected phase volume," $\rho(E)=P_{0}\left(E, r_{\mathrm{ch}}^{(p p)}, 1\right)$. Finally, a treatment of this density in the spirit of the MigdalWatson approximation can be found in Ref. [50].

It should be noted that in the diproton picture, the information about the nuclear interior is lost (except the spectroscopic 
information contained in $\gamma_{p p}$ ) and we can obtain information only about the peculiarities of the final-state interaction from measurements of the proton correlations.

From the theoretical side it was demonstrated in Ref. [23] that the diproton model is typically used in an inconsistent way in terms of the accepted parametrizations for the diproton channel radius $r_{\mathrm{ch}}^{(d p)}$. Correctly applied, the diproton model provides unrealistically small values of the width.

From the experimental side, one can see that Eq. (31) provides a single broad peak in the energy distribution in the "T" system, while double-hump structure is present in the data. This problem of the diproton model has been pointed already in Ref. [44]. Moreover, Eq. (31) provides an angular-independent decay probability in the " $T$ " system. The observed angular distributions in the "T" system [Fig. 18(a), Fig. 21 (left column)] thus demonstrate one more time that the $2 p$ decay process cannot be reasonably approximated as a diproton decay.

\section{Quasiclassical-simultaneous-emission model}

Another frequently used approximation for $2 p$ decays is the simultaneous-emission model, which neglects the $p$ - $p$ interaction and considers the decay via the low-energy tails of the resonances in the core- $p$ subsystem. The formula for the width (including preexponent) in this approximation was derived in Ref. [55] and then in more details in Ref. [24]:

$$
\begin{aligned}
\frac{d \Gamma}{d \varepsilon}= & \frac{E_{T}\left\langle V_{3}\right\rangle^{2}}{2 \pi} \frac{\Gamma_{p_{1}}\left(\varepsilon E_{T}\right)}{\left(\varepsilon E_{T}-E_{p_{1}}\right)^{2}+\Gamma_{p_{1}}\left(\varepsilon E_{T}\right)^{2} / 4} \\
& \times \frac{\Gamma_{p_{2}}\left((1-\varepsilon) E_{T}\right)}{\left((1-\varepsilon) E_{T}-E_{p_{2}}\right)^{2}+\Gamma_{p_{2}}\left((1-\varepsilon) E_{T}\right)^{2} / 4} \\
\Gamma_{p_{i}}\left(E_{i}\right)= & 2 \frac{\theta_{i}^{2}}{2 M_{i} r_{\mathrm{ch} i}^{2}} P_{l_{i}}\left(E_{i}, r_{\mathrm{ch} i}, Z_{i}\right) . \\
\left\langle V_{3}\right\rangle^{2}= & D_{3}\left(E_{T}-E_{p 1}-E_{p 2}\right)^{2},
\end{aligned}
$$

In this equation, $E_{p_{i}}$ and $\Gamma_{p_{i}}$ are parameters of the groundstate resonance in the core $+p_{i}$ subsystem and $D_{3} \approx 1$. In the literature (e.g., Refs. [56,57]) a version of Eq. (32) is used that can be obtained by letting one of the resonance energies $E_{p_{i}}$ tend to infinity. This is not a reasonable assumption as, for $0^{+}$

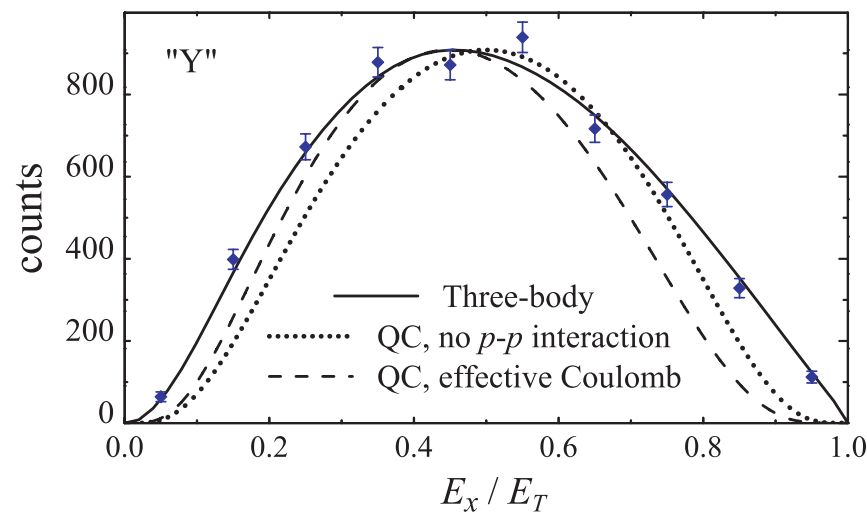

FIG. 22. (Color online) The energy distribution for ${ }^{6} \mathrm{Be}$ in the "Y" system compared with quasiclassical predictions [Eq. (32)].
TABLE V. Widths of the ${ }^{6}$ Be g.s. calculated in the simultaneous emission model [Eq. (32)].

\begin{tabular}{lllllr}
\hline \hline Model & $r_{\mathrm{ch} 1}(\mathrm{fm})$ & $\theta_{1}^{2}$ & $r_{\mathrm{ch} 2}(\mathrm{fm})$ & $\theta_{2}^{2}$ & $\begin{array}{c}\Gamma \\
(\mathrm{keV})\end{array}$ \\
\hline No $p-p$ & 2.39 & 1.585 & 2.39 & 1.585 & 20.4 \\
No $p-p$ & 4.0 & 1.305 & 4.0 & 1.305 & 35.1 \\
Effective & 2.39 & 1.585 & 2.39 & 1.945 & 14.7 \\
Effective & 4.0 & 1.305 & 4.0 & 1.505 & 25.9 \\
\hline \hline
\end{tabular}

ground-state decays, both of the lowest resonances $E_{p_{i}}$ in the subsystems have the same energy.

In the derivation of Eq. (32) in Ref. [24], two reasonable assumptions about subsystems were considered. (i) We can neglect the interaction between protons. Then, the "charges" for both subsystems should be taken as $Z_{i}=Z_{\text {core }}$. This is the "no $p$ - $p$ interaction" case in Fig. 22 where the predicted $E_{x} / E_{T}$ profile in the "Y" system does not reproduce the visible shift of the experimental profile to the left. (ii) We can consider one of the subsystems as an effective particle with charge $Z_{2}=$ $Z_{\text {core }}+1$ (while $Z_{1}=Z_{\text {core }}$ ). In this "effective Coulomb" case, the shift of the profile to the left is reproduced correctly, but the wings of the distribution are still wrong.

The results of the width calculations are provided in Table V. The dimensionless reduced widths $\theta^{2}$ are fitted in such a way that they provide reasonable properties for the ${ }^{5} \mathrm{Li}$ subsystem. The $3 / 2^{-}$ground state is located at $E_{2 r}=$ $2.08 \mathrm{MeV}$ with $\Gamma=2.11 \mathrm{MeV}$ [15]. The calculated widths are 2.5-6 times smaller than the experimental value. There is a considerable "unphysical" sensitivity of the width, defined by Eq. (32), to the channel radius, but even this is not sufficient to obtain a reasonable value by fine tuning $r_{\mathrm{ch} i}$.

The reason for disagreement between the quasiclassical predictions and the experiment is the same for both the width and for the energy distributions. This can be demonstrated with the help of Table I. One can readily see that in the interior of ${ }^{6} \mathrm{Be}$, the $\left[p^{2}\right]$ configuration is dominating (its weight is provided with a good accuracy by the weights $N_{i}$ of the $K=$ $2, S=0,1$ configurations). However, in the asymptotic region, the $\left[s^{2}\right]$ configuration becomes very important (its contribution corresponds to the partial width of the $K=0$ configuration). The influence of this configuration increases the $2 p$ penetration rate (due to the lower centrifugal barrier) and simultaneously provides somewhat broader energy distributions in the "Y"

TABLE VI. Weights of the shell-model-like configurations [ $\left.\ell^{2}\right]$ in the ${ }^{6} \mathrm{He}$ and ${ }^{6} \mathrm{Be}$ g.s. WFs in percentages for the Jacobi "Y" system. The normalizations of the ${ }^{6} \mathrm{Be}$ components are found for integration radius $\rho_{\text {int }}=12.5 \mathrm{fm}$.

\begin{tabular}{lrrrrrrr}
\hline \hline$\left[\ell^{2}\right]$ & \multicolumn{3}{c}{${ }^{6} \mathrm{He}$} & & \multicolumn{4}{c}{${ }^{6} \mathrm{Be}$} \\
\cline { 2 - 3 } & \multicolumn{1}{c}{$\mathrm{P} 1$} & \multicolumn{1}{c}{$\mathrm{P} 2$} & \multicolumn{1}{c}{$\mathrm{P} 3$} & & $\mathrm{P} 1$ & \multicolumn{1}{c}{$\mathrm{P} 2$} & $\mathrm{P} 3$ \\
\hline$\left[s^{2}\right]$ & 8.11 & 8.58 & 8.35 & & 10.54 & 11.15 & 10.84 \\
{$\left[p^{2}\right]$} & 90.91 & 90.30 & 90.37 & & 87.98 & 87.18 & 87.17 \\
{$\left[d^{2}\right]$} & 0.47 & 0.53 & 0.61 & & 0.69 & 0.77 & 0.95 \\
{$\left[f^{2}\right]$} & 0.41 & 0.43 & 0.50 & & 0.60 & 0.65 & 0.77 \\
\hline \hline
\end{tabular}


system. There is no way to consider the contribution of the $\left[s^{2}\right]$ configuration within approximation of Eq. (32), which presumes resonant continuum in both core- $p$ subsystems.

The three-body calculations of Sec. II provide a perfect fit to the experimental data in Fig. 22. The quasiclassical estimates can successfully mimic the overall picture (namely the width can be predicted within an order of the magnitude and the profile of the energy distribution in "Y" system can be reasonable) but fails when high-quality data are available.

\section{CONCLUSIONS}

The first detailed studies of the correlations from the decay of the ${ }^{6} \mathrm{Be}$ g.s. are performed both experimentally and theoretically. We have found that certain correlations (namely energy correlation between two protons in the "T" system and angular correlations in "Y" Jacobi system) are quite sensitive to the details of the structure and interactions.

Experimentally ${ }^{6} \mathrm{Be}$ fragments are produced from the $\alpha$ decay of ${ }^{10} \mathrm{C}$ excited states formed by inelastically scattering a ${ }^{10} \mathrm{C}$ beam off of $\mathrm{Be}$ and $\mathrm{C}$ targets. The $\alpha+2 p$ decay products as well as the initially emitted $\alpha$ particle were detected in a Si array with good position and energy resolution. The experimentally measured correlations between the ${ }^{6} \mathrm{Be}$ g.s. decay products and the theoretical predictions were found to be in good agreement.

We have demonstrated that the relative sensitivity of the continuum observables (including correlation patterns) to the details of the interactions is higher in the decay of ${ }^{6} \mathrm{Be}$ compared to the corresponding sensitivity of typical observables in ${ }^{6} \mathrm{He}$. We argue that further highly detailed studies of the correlations in the decay of ${ }^{6} \mathrm{Be}$ could provide better access to the properties of the $A=6$ isobars (and thus to the halo properties of the ${ }^{6} \mathrm{He}$ nucleus) than the direct studies of ${ }^{6} \mathrm{He}$ halo properties.

\section{ACKNOWLEDGMENTS}

This work was supported by the US Department of Energy, Division of Nuclear Physics under Grants DE-FG0287ER-40316, DE-FG02-93ER40773, DE-FG02-04ER413, and DE-FG52-06NA26207. L.V.G. acknowledges the support from Deutsche Forschungsgemeinschaft Grant 436 RUS 113/907/0-1, Russian Foundation for Basic Research Grants RFBR 08-02-00892, RFBR 08-02-00089-a, and Russian Ministry of Industry and Science Grant NS-3004.2008.2.
[1] V. I. Goldansky, Nucl. Phys. 19, 482 (1960).

[2] M. Pfutzner, E. Badura, C. Bingham, B. Blank, M. Chartier, H. Geissel, J. Giovinazzo, L. V. Grigorenko, R. Grzywacz, M. Hellstrom, Z. Janas, J. Kurcewicz, A. S. Lalleman, C. Mazzocchi, I. Mukha, G. Munzenberg, C. Plettner, E. Roeckl, K. P. Rykaczewski, K. Schmidt, R. S. Simon, M. Stanoiu, and J.-C. Thomas, Eur. Phys. J. A 14, 279 (2002).

[3] J. Giovinazzo, B. Blank, M. Chartier, S. Czajkowski, A. Fleury, M. J. Lopez Jimenez, M. S. Pravikoff, J.-C. Thomas, F. de Oliveira Santos, M. Lewitowicz, V. Maslov, M. Stanoiu, R. Grzywacz, M. Pfutzner, C. Borcea, and B. A. Brown, Phys. Rev. Lett. 89, 102501 (2002).

[4] B. Blank, A. Bey, G. Canchel, C. Dossat, A. Fleury, J. Giovinazzo, I. Matea, N. Adimi, F. De Oliveira, I. Stefan, G. Georgiev, S. Grevy, J. C. Thomas, C. Borcea, D. Cortina, M. Caamano, M. Stanoiu, F. Aksouh, B. A. Brown, F. C. Barker, and W. A. Richter, Phys. Rev. Lett. 94, 232501 (2005).

[5] I. Mukha, K. Sümmerer, L. Acosta, M. A. G. Alvarez, E. Casarejos, A. Chatillon, D. Cortina-Gil, J. Espino, A. Fomichev, J. E. García-Ramos, H. Geissel, J. Gómez-Camacho, L. Grigorenko, J. Hofmann, O. Kiselev, A. Korsheninnikov, N. Kurz, Yu. Litvinov, I. Martel, C. Nociforo, W. Ott, M. Pfutzner, C. Rodríguez-Tajes, E. Roeckl, M. Stanoiu, H. Weick, and P. J. Woods, Phys. Rev. Lett. 99, 182501 (2007).

[6] C. Dossat, A. Bey, B. Blank, G. Canchel, A. Fleury, J. Giovinazzo, I. Matea, F. de Oliveira Santos, G. Georgiev, S. Grèvy, I. Stefan, J. C. Thomas, N. Adimi, C. Borcea, D. Cortina Gil, M. Caamano, M. Stanoiu, F. Aksouh, B. A. Brown, and L. V. Grigorenko, Phys. Rev. C 72, 054315 (2005).

[7] K. Miernik, W. Dominik, Z. Janas, M. Pfützner, L. Grigorenko, C. R. Bingham, H. Czyrkowski, M. Cwiok, I. G. Darby, R. Dabrowski, T. Ginter, R. Grzywacz, M. Karny, A. Korgul, W. Kusmierz, S. N. Liddick, M. Rajabali, K. Rykaczewski, and A. Stolz, Phys. Rev. Lett. 99, 192501 (2007).
[8] I. Mukha, L. Grigorenko, K. Sümmerer, L. Acosta, M. A. G. Alvarez, E. Casarejos, A. Chatillon, D. Cortina-Gil, J. M. Espino, A. Fomichev, J. E. Garcia-Ramos, H. Geissel, J. Gomez-Camacho, J. Hofmann, O. Kiselev, A. Korsheninnikov, N. Kurz, Yu. Litvinov, I. Martel, C. Nociforo, W. Ott, M. Pfützner, C. Rodriguez-Tajes, E. Roeckl, M. Stanoiu, H. Weick, and P. J. Woods, Phys. Rev. C 77, 061303(R) (2008).

[9] K. Mercurio, R. J. Charity, R. Shane, L. G. Sobotka, J. M. Elson, M. Famiano, A. H. Wuosmaa, A. Banu, C. Fu, L. Trache, R. E. Tribble, and A. M. Mukhamedzhanov, Phys. Rev. C 78, 031602(R) (2008); R. J. Charity, T. D. Wiser, K. Mercurio, R. Shane, L. G. Sobotka, A. H. Wuosmaa, A. Banu, L. Trache, and R. E. Tribble, Phys. Rev. C 80, 024306 (2009).

[10] I. Mukha, E. Roeckl, L. Batist, A. Blazhev, J. Döring, H. Grawe, L. Grigorenko, M. Huyse, Z. Janas, R. Kirchner, M. La Commara, C. Mazzocchi, S. L. Tabor, and P. Van Duppen, Nature 439, 298 (2006).

[11] O. L. Pechenaya, C. J. Chiara, D. G. Sarantites, W. Reviol, R. J. Charity, M. P. Carpenter, R. V. F. Janssens, T. Lauritsen, C. J. Lister, D. Seweryniak, S. Zhu, L.-L. Andersson, E. K. Johansson, and D. Rudolph, Phys. Rev. C 76, 011304(R) (2007).

[12] A. Kankainen, V.-V. Elomaa, L. Batist, S. Eliseev, T. Eronen, U. Hager, J. Hakala, A. Jokinen, I. D. Moore, Yu. N. Novikov, H. Penttilä, A. Popov, S. Rahaman, S. Rinta-Antila, J. Rissanen, A. Saastamoinen, D. M. Seliverstov, T. Sonoda, G. Vorobjev, C. Weber, and J. Äystö, Phys. Rev. Lett. 101, 142503 (2008).

[13] O. V. Bochkarev, A. A. Korsheninnikov, E. A. Kuz'min, I. G. Mukha, L. V. Chulkov, and G. B. Yan'kov, Yad. Fiz. 55, 1729 (1992) [Sov. J. Nucl. Phys. 55, 955 (1992)].

[14] L. V. Grigorenko, T. D. Wiser, K. Miernik, R. J. Charity, M. Pfützner, A. Banu, C. R. Bingham, M. Cwiok, I. G. Darby, W. Dominik, J. M. Elson, T. Ginter, R. Grzywacz, Z. Janas, M. Karny, A. Korgul, S. N. Liddick, K. Mercurio, M. Rajabali, K. Rykaczewski, R. Shane, L. G. Sobotka, A. Stolz, L. Trache, 
R. E. Tribble, A. H. Wuosmaa, and M. V. Zhukov, Phys. Lett. B677, 30 (2009).

[15] D. R. Tilley, C. M. Cheves, J. L. Godwin, G. M. Hale, H. M. Hofmann, J. H. Kelley, C. G. Sheu, and H. R. Weller, Nucl. Phys. A708, 3 (2002).

[16] L. V. Grigorenko, R. C. Johnson, I. G. Mukha, I. J. Thompson, and M. V. Zhukov, Phys. Rev. Lett. 85, 22 (2000).

[17] L. V. Grigorenko, R. C. Johnson, I. G. Mukha, I. J. Thompson, and M. V. Zhukov, Phys. Rev. C 64, 054002 (2001).

[18] L. V. Grigorenko and M. V. Zhukov, Phys. Rev. C 68, 054005 (2003).

[19] L. V. Grigorenko, I. G. Mukha, I. J. Thompson, and M. V. Zhukov, Phys. Rev. Lett. 88, 042502 (2002).

[20] L. V. Grigorenko, R. C. Johnson, I. G. Mukha, I. J. Thompson, and M. V. Zhukov, Eur. Phys. J. A 15, 125 (2002).

[21] L. V. Grigorenko, I. G. Mukha, and M. V. Zhukov, Nucl. Phys. A713, 372 (2003) [Erratum-ibid. A740, 401 (2004)].

[22] L. V. Grigorenko, I. G. Mukha, and M. V. Zhukov, Nucl. Phys. A714, 425 (2003).

[23] L. V. Grigorenko and M. V. Zhukov, Phys. Rev. C 76, 014008 (2007).

[24] L. V. Grigorenko and M. V. Zhukov, Phys. Rev. C 76, 014009 (2007).

[25] G. E. Brown and A. D. Jackson, The Nucleon-Nucleon Interaction (North Holland, Amsterdam, 1976).

[26] D. Gogny, P. Pires, R. de Tourreil, Phys. Lett. B32, 591 (1970).

[27] S. Sack, L. C. Biedenharn, and G. Breit, Phys. Rev. 93, 321 (1954).

[28] B. V. Danilin, M. V. Zhukov, S. N. Ershov, F. A. Gareev, R. S. Kurmanov, J. S. Vaagen, and J. M. Bang, Phys. Rev. C 43, 2835 (1991).

[29] M. S. Golovkov, L. V. Grigorenko, A. S. Fomichev, Yu. Ts. Oganessian, Yu. I. Orlov, A. M. Rodin, S. I. Sidorchuk, R. S. Slepnev, S. V. Stepantsov, G. M. Ter-Akopian, and R. Wolski, Phys. Lett. B588, 163 (2004).

[30] O. V. Bochkarev, A. A. Korsheninnikov, E. A. Kuz'min, I. G. Mukha, A. A. Ogloblin, L. V. Chulkov, and G. B. Yan'kov, JETP Lett. 40, 969 (1984).

[31] O. V. Bochkarev, L. V. Chulkov, A. A. Korsheninnikov, E. A. Kuzmin, I. G. Mukha, and G. B. Yankov, Nucl. Phys. A505, 215 (1989).

[32] B. V. Danilin, M. V. Zhukov, A. A. Korsheninnikov, L. V. Chulkov, and V. D. Efros, Yad. Fiz. 46, 427 (1987) [Sov. J. Nucl. Phys. 46, 225 (1987)].

[33] P. Egelhof, Prog. Part. Nucl. Phys. 46, 307 (2001).

[34] A. Ozawa, T. Sizuki, and I. Tanihata, Nucl. Phys. A693, 32 (2001).

[35] L.-B. Wang, P. Mueller, K. Bailey, G. W. F. Drake, J. P. Greene, D. Henderson, R. J. Holt, R. V. F. Janssens, C. L. Jiang,
Z.-T. Lu, T. P. O'Connor, R. C. Pardo, K. E. Rehm, J. P. Schiffer, and X. D. Tang, Phys. Rev. Lett. 93, 142501 (2004).

[36] P. Mueller, I. A. Sulai, A. C. C. Villari, J. A. Alcantara-Nunez, R. Alves-Conde, K. Bailey, G. W. F. Drake, M. Dubois, C. Eleon, G. Gaubert, R. J. Holt, R. V. F. Janssens, N. Lecesne, Z.-T. Lu, T. P. O'Connor, M.-G. Saint-Laurent, J.-C. Thomas, and L.-B. Wang, Phys. Rev. Lett. 99, 252501 (2007).

[37] B. V. Danilin and M. V. Zhukov, Yad. Fiz. 56, 67 (1993) [Phys. At. Nucl. 56, 460 (1993)].

[38] A. Csoto, Phys. Rev. C 49, 3035 (1994).

[39] V. Vasilevsky, A. V. Nesterov, F. Arickx, and J. Broeckhove, Phys. Rev. C 63, 034607 (2001).

[40] P. Descouvemont, E. Tursunov, and D. Baye, Nucl. Phys. A765, 370 (2006).

[41] M. Gulyamov, B. V. Rybakov, and V. A. Sidarov, Sov. Phys. JETP 17, 1230 (1963) [Zh. Eksp. Teor. Fiz. 44, 1829 (1963)].

[42] W. Whaling, Phys. Rev. 150, 836 (1966).

[43] J. L. Honsaker, Nucl. Phys. A90, 545 (1967).

[44] D. F. Geesaman, R. L. McGrath, P. M. S. Lesser, P. P. Urone, and B. VerWest, Phys. Rev. C 15, 1835 (1977).

[45] R. E. Tribble, R. H. Burch, and C. A. Gagliardi, Nucl. Instrum. Methods A 285, 411 (1989).

[46] M. S. Wallace, M. A. Famiano, M.-J. van Goethem, A. M. Rogers, W. G. Lynch, J. Clifford, F. Delaunay, J. Lee, S. Labostov, M. Mocko, L. Morris, A. Moroni, B. E. Nett, D. J. Oostdyk, R. Krishnasamy, M. B. Tsang, R. T. de Souza, S. Hudan, L. G. Sobotka, R. J. Charity, J. Elson, and G. L. Engel, Nucl. Instrum. Methods A 583, 302 (2007).

[47] J. F. Ziegler, J. P. Biersack, and U. Littmark, The Stopping and Range of Ions in Solids (Pergamon Press, New York, 1985). The code SRIM can be found at http://www.srim.org.

[48] R. Anne, J. Herault, R. Bimbot, H. Gauvin, G. Bastin, and F. Hubert, Nucl. Instrum. Methods B 34, 295 (1988).

[49] V. I. Goldansky, Nucl. Phys. 27, 648 (1961).

[50] F. C. Barker, Phys. Rev. C 63, 047303 (2001).

[51] B. A. Brown, F. C. Barker, and D. J. Millener, Phys. Rev. C 65, 051309(R) (2002).

[52] F. C. Barker, Phys. Rev. C 66, 047603 (2002).

[53] F. C. Barker, Phys. Rev. C 68, 054602 (2003).

[54] B. A. Brown and F. C. Barker, Phys. Rev. C 67, 041304(R) (2003).

[55] V. M. Galitsky and V. F. Cheltsov, Nucl. Phys. 56, 86 (1964).

[56] R. A. Kryger, A. Azhari, M. Hellstrom, J. H. Kelley, T. Kubo, R. Pfaff, E. Ramakrishnan, B. M. Sherrill, M. Thoennessen, S. Yokoyama, R. J. Charity, J. Dempsey, A. Kirov, N. Robertson, D. G. Sarantites, L. G. Sobotka, and J. A. Winger, Phys. Rev. Lett. 74, 860 (1995).

[57] F. C. Barker, Phys. Rev. C 59, 535 (1999). 